\title{
乌s \\ Experimental study of current loss and plasma formation in the $Z$ machine post-hole convolute
}

\author{
M. R. Gomez, ${ }^{1, *}$ R. M. Gilgenbach, ${ }^{2}$ M. E. Cuneo, ${ }^{1}$ C. A. Jennings, ${ }^{1}$ R. D. McBride,,${ }^{2, \dagger}$ \\ E. M. Waisman, ${ }^{1}$ B. T. Hutsel, ${ }^{1}$ W. A. Stygar, ${ }^{1}$ D. V. Rose, ${ }^{3}$ and Y. Maron ${ }^{4}$ \\ ${ }^{1}$ Sandia National Laboratories, Albuquerque, New Mexico 87185, USA \\ ${ }^{2}$ Nuclear Engineering and Radiological Sciences Department, \\ University of Michigan, Ann Arbor, Michigan 48109, USA \\ ${ }^{3}$ Voss Scientific, LLC, Albuquerque, New Mexico 87108, USA \\ ${ }^{4}$ Department of Physics, Weizmann Institute of Science, Rehovot 76100, Israel
}

(Received 23 August 2016; published 24 January 2017)

\begin{abstract}
The $Z$ pulsed-power generator at Sandia National Laboratories drives high energy density physics experiments with load currents of up to 26 MA. $Z$ utilizes a double post-hole convolute to combine the current from four parallel magnetically insulated transmission lines into a single transmission line just upstream of the load. Current loss is observed in most experiments and is traditionally attributed to inefficient convolute performance. The apparent loss current varies substantially for z-pinch loads with different inductance histories; however, a similar convolute impedance history is observed for all load types. This paper details direct spectroscopic measurements of plasma density, temperature, and apparent and actual plasma closure velocities within the convolute. Spectral measurements indicate a correlation between impedance collapse and plasma formation in the convolute. Absorption features in the spectra show the convolute plasma consists primarily of hydrogen, which likely forms from desorbed electrode contaminant species such as $\mathrm{H}_{2} \mathrm{O}, \mathrm{H}_{2}$, and hydrocarbons. Plasma densities increase from $1 \times 10^{16} \mathrm{~cm}^{-3}$ (level of detectability) just before peak current to over $1 \times 10^{17} \mathrm{~cm}^{-3}$ at stagnation (tens of ns later). The density seems to be highest near the cathode surface, with an apparent cathode to anode plasma velocity in the range of $35-50 \mathrm{~cm} / \mu \mathrm{s}$. Similar plasma conditions and convolute impedance histories are observed in experiments with high and low losses, suggesting that losses are driven largely by load dynamics, which determine the voltage on the convolute.
\end{abstract}

DOI: 10.1103/PhysRevAccelBeams.20.010401

\section{INTRODUCTION}

High energy density physics experiments conducted on Sandia's $Z$ machine $[1,2]$ range from dynamic material property measurements $[3,4]$ to inertial confinement fusion (ICF) experiments [5-7] to laboratory astrophysics [8,9]. These experiments are $\mathrm{cm}$-scale, $100 \mathrm{~ns}-1 \mu$ s duration, and must be conducted in vacuum. As a result, the electrical pulse generated in $Z$ 's oil section and compressed in the water section must pass through an insulator stack which acts as the water-vacuum boundary [1]. In order to reduce inductance within the vacuum section, four parallel magnetically insulated transmission lines (MITL) are used to

\footnotetext{
*Portions of this work were conducted while a student in the Nuclear Engineering and Radiological Sciences Department at the University of Michigan, Ann Arbor, MI.

This work was conducted while a staff member in the Pulsed Power Sciences Center at Sandia National Laboratories.

Published by the American Physical Society under the terms of the Creative Commons Attribution 4.0 International license. Further distribution of this work must maintain attribution to the author(s) and the published article's title, journal citation, and DOI.
}

transfer the pulse from the stack towards the on-axis load [1]. At a radius of $7.6 \mathrm{~cm}$, the currents from the four MITLs are combined and fed into a single anode-cathode (A-K) gap using a double post-hole convolute [10,11], as shown in Fig. 1.

A post-hole convolute is a device in which the currents from two transmission lines are combined by connecting the two anodes with a post through a hole in the common cathode. Often multiple post holes are used in parallel to reduce the inductance of the device (e.g., Saturn [14] uses six post holes [10]; the $Z$ machine uses 12). Post-hole convolutes can be stacked to combine current from an even number of transmission lines. The $Z$ machine has four transmission lines, which requires a double post-hole convolute.

$Z$ is capable of delivering a 27 MA pulse with a sub$100 \mathrm{~ns}$ rise time $(10 \%-90 \%)$ to the vacuum insulator stack [1]. In many $Z$ experiments, a measurable portion ( $>1 \mathrm{MA}$ ) of the current delivered to the stack does not reach the load [15-21]. The reduction in load current is typically attributed to inefficient convolute performance, which has been described in circuit modeling as a time-dependent shunt impedance [18,19]; this is sometimes described as $Z$-flow [15]. The shunt impedance has the form of a rapid 

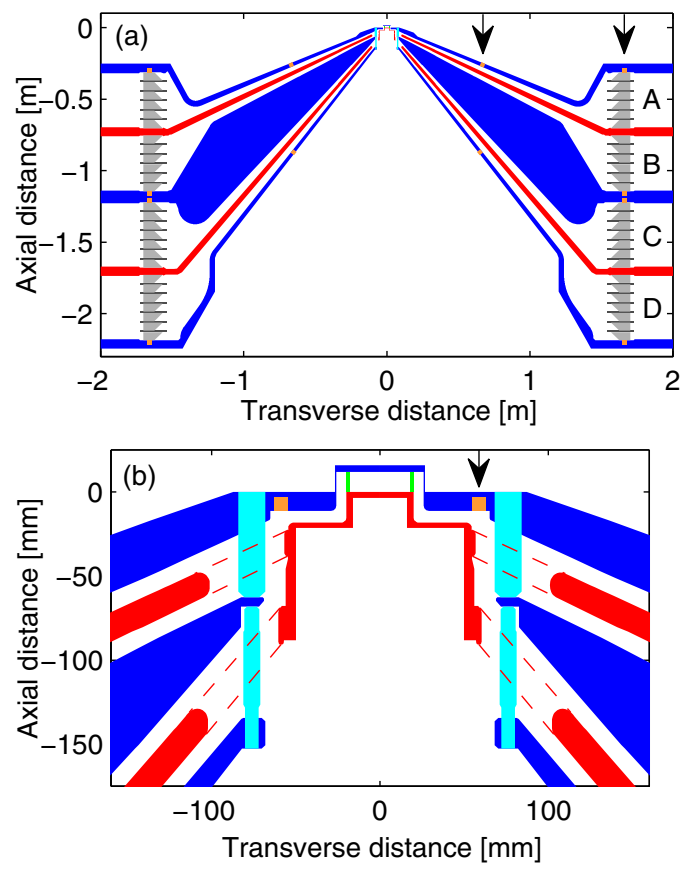

FIG. 1. (a) An $R-Z$ cross section of the vacuum section and insulator stack of the $Z$ machine [1]. The vacuum section contains four outer-magnetically insulated transmission lines (MITLs) labeled A through D with anodes shown in blue and cathodes in red. Arrows indicate the radial location of the outer-MITL B-dot monitors (at $R=0.66 \mathrm{~m}$ on the $\mathrm{A}$ and $\mathrm{D}$ levels) and the stack B-dot and D-dot monitors (at $R=1.66 \mathrm{~m}$ on the $\mathrm{A}, \mathrm{B}, \mathrm{C}$, and D levels). (b) An $R-Z$ cross section of the double post-hole convolute with the z-pinch dynamic-hohlraum [12,13] load geometry. Anodes are shown in blue, convolute anode posts in cyan, cathodes in red, and holes in the cathode are represented by dashed red lines. An arrow indicates the radial location of the inner-MITL B-dot monitors (tan squares). The inner-MITL region $(R<58 \mathrm{~mm})$ varies with load type.

decay ( $\sim 10 \mathrm{~ns}$ ) from high resistance $(>10 \mathrm{ohms})$ to a nearly constant, relatively low value $(<1 \mathrm{ohm})$ for all load types [20]. The timing of the impedance collapse and functional form of the impedance for each experimental configuration is empirically determined and then applied to subsequent identical experiments.

Due to the complex geometry of Z's double post-hole convolute, one extended and 36 localized magnetic nulls form (see Fig. 5 of Ref. [22]); initially these nulls were believed to be the source of current loss in the convolute. Numerous particle-in-cell (PIC) and hybrid fluid-PIC simulations of the $Z$ and $Z R$ convolutes have been conducted to examine the observed current loss [11,22-28], and these studies show that electron flow current crossing the anode-cathode gap through the magnetic nulls in the convolute cannot account for the majority of observed current loss $[22,25,26]$. Simulations that only include electron emission from the cathode and ion emission from the anode also significantly underestimate the current loss; electrode plasma formation must be included in simulations to match the experimentally observed losses [22,25,26]. It is worth noting that negative ions have been observed in other pulsed power drivers [29], but they have not been included in these simulations of the convolute. It is not clear what impact negative ions have on current loss in the convolute at this time.

These simulations assume that hydrogen desorbs from the electrodes and is ionized immediately in the first cell adjacent to the conducting surface [22]. Once ionized, the plasma flows in all directions, but preferentially along magnetic field lines. Simulations indicate that plasma collects in the cathode hole on the downstream side of the upper convolute posts [22,28], as shown in Fig. 2 (see Fig. 4 of Ref. [28] for a more detailed image). This plasma is sufficiently dense to act as an extension of the cathode, which allows space charge limited emission to occur with a smaller anode-cathode gap, thus enhancing the current loss [22]. The hydrogen desorption rate in these simulations was not measured experimentally or derived theoretically, rather the rate was varied until the simulated current traces matched the experimental measurements [22]. The optimally tuned desorption rate was 0.0075 monolayers/ns of hydrogen, or 0.75 monolayers in $100 \mathrm{~ns}$. One monolayer is a neutral areal density of approximately $1 \times 10^{15} \mathrm{~cm}^{-2}$. This desorption rate provided agreement between the measured and simulated loss for several convolute geometries [22,25], but the
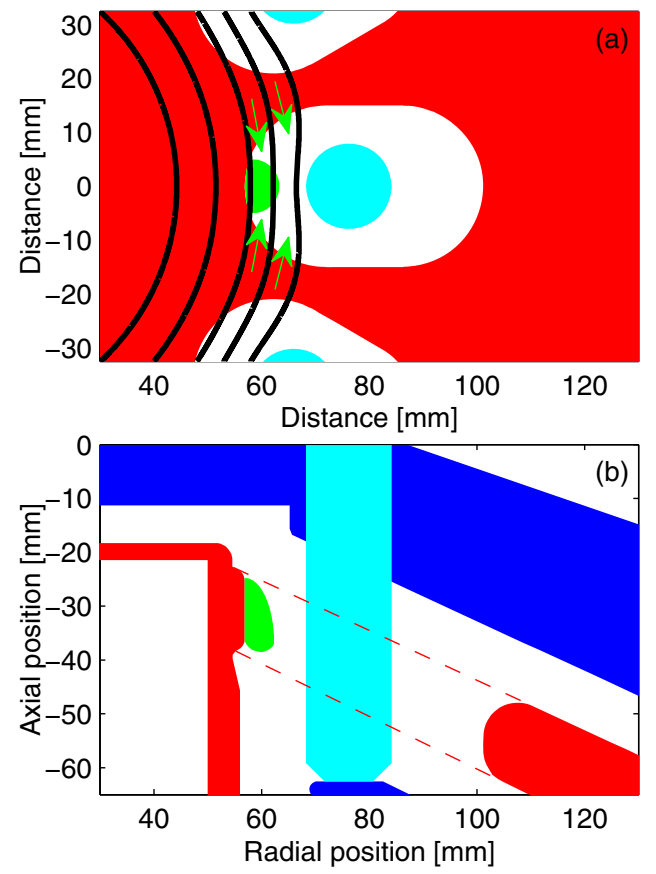

FIG. 2. (a) An $R-\theta$ cross section of the upper post-hole convolute. Magnetic field lines are shown in black. Cartoon representations of plasma formation and the collection sites [22] on the downstream side of the convolute are shown in green. (b) An $R-Z$ cross section of the convolute showing the plasma collection site as predicted in simulations $[22,25]$. 
parameters of the resultant plasma have not been experimentally verified.

More recently, simulations of a simplified convolute geometry have been conducted to understand the impact of varying a number of parameters including post radius, number of posts, and anode-cathode gap spacing [27,28]. These simulations used a fixed, relatively high-impedance load to accentuate the loss mechanisms by increasing the voltage in the MITL-convolute system. As demonstrated previously, these simulations showed that plasma formation must be included to produce substantial losses [28]. Although these simulations showed plasma collecting on the downstream side of the cathode holes, this was not the only important source of loss. In the simulations, electron flow from the outer-MITLs lost at the convolute was also critical. The enhanced energy deposition in the convolute electrodes from the electron flow increased plasma formation, which amplified local electron loss [28]. These simulations indicate that the convolute can only be understood in the context of a coupled MITL-convolute system.

In both experiments and simulations, the efficiency of current addition in the convolute is observed to decrease with increasing electrical power [11,22-28]. Losses in the $Z$ convolute prior to the refurbishment project for low-impedance $z$-pinch loads were as high as $1.5 \mathrm{MA}$ out of 18.5 MA [22], but after refurbishment, the stored energy of $Z$ doubled, and the convolute losses increased to 3 MA out of 23 MA for similar low-impedance loads [25]. This is significant because designs for new, highercurrent (50 + MA) generators such as Baikal [30-32] and $Z$-Next $[33,34]$ roughly quadruple the electrical power of $Z$, and their designs include a triple post-hole convolute to add current from six MITL levels into a single final power feed. In order to move forward with future highercurrent accelerators, a validated understanding of convolute science is required such that new convolute designs, which increase convolute efficiency, can be developed.

This paper describes a series of experimental measurements of current flow through, and plasma formation in, the $Z$ machine double post-hole convolute for a variety of experimental load types. These measurements indicate a correlation between plasma formation and impedance collapse, and they qualitatively support the findings of numerical simulations, which suggest that plasma on the downstream side of the convolute post can lead to current loss on $Z$. Furthermore, the measurements indicate current loss is driven primarily by load dynamics, which determine the voltage on the convolute. Section II discusses the determination of current loss via electrical measurements. Section III provides details on the visible spectroscopy measurements, including the plasma parameter inference methodology. Section IV presents conclusions and future work.

\section{ELECTRICAL MEASUREMENTS}

Electrical diagnostics are located throughout the $Z$ machine vacuum region to monitor current and voltage [35]. B-dot monitors are used to determine the current, and D-dot monitors are used to determine the voltage. B-dots at the insulator stack $(r=166 \mathrm{~cm})$ monitor each of the four transmission-line levels, B-dots in the outer-MITLs $(r=66 \mathrm{~cm})$ monitor only the A and $\mathrm{D}$ transmission lines, and B-dots in the inner-MITL $(r=5.9 \mathrm{~cm})$ monitor the single final-anode-cathode gap (see Fig. 1). Up to 18 differential B-dot pairs per level, fielded roughly every 20 degrees around the azimuth, are used to determine the current at the stack, up to nine differential B-dot pairs per level are fielded every 40 degrees in the outer-MITLs, and 2-10 single B-dots are fielded in the inner-MITL, often in opposite polarity pairs. Up to $18 \mathrm{D}$-dot monitors are fielded in each of the four transmission-line levels at the insulator stack. The measured signals are combined to determine: (1) the current at each level of the insulator stack, (2) the voltage at each level of the insulator stack, (3) the total stack current, (4) the average stack voltage, (5) the estimated total outer-MITL current, and (6) the inner-MITL current.

Note that the current inferred at each B-dot location is the anode current, which is equal to the sum of the current trapped within the cathode electrode and the electron flow current in the A-K gap. The flow current has not yet been lost to the anode, but the majority of the flow current is not expected to retrap in the cathode $[27,36]$. There is no flow current at the insulator stack B-dot position because these monitors are located just outside the vacuum region, so the measured anode current and the trapped cathode current are equivalent. At the inner-MITL location, simulations indicate the electron flow current can be several MA [28]. In the following arguments, current refers to the anode current, which is an upper bound on the trapped cathode current at that location.

Circuit models of the $Z$ vacuum section have been developed to determine the expected current delivery to other regions of the transmission line assuming lossless propagation of the measured current and voltage $[18,37,38]$. For example, the current measured at the stack can be propagated to the location of the outer-MITL current measurements, which allows a direct comparison between the two currents in order to quantify the reduction in current delivery. The difference between the current losslessly propagated to a point and the current measured at that point is referred to as the shunt current in that region of the MITLs. Similarly, the voltage measured at the stack can be translated to other points in the MITLs using this circuit model $[18,37,38]$. On several experiments, an inductive voltage monitor was fielded at the convolute, which confirmed that the transmission-line model correctly determined the convolute voltage [39]. 
It is worth emphasizing that there is a difference between the shunt current and the amount of current that would be recovered in a lossless system [20,27]. Because current loss provides a low-impedance path in parallel with the load impedance, the total impedance of the system decreases with increasing loss. Thus for a given driving voltage pulse, more total current is delivered by the accelerator to the MITL-convolute-load system, even though less current reaches the load. Simulations show that the current that would be recovered in a lossless system is generally about half of the shunt current [20,27].

The refurbished $Z$ accelerator was designed to drive z-pinch dynamic hohlraum (ZPDH) [12,13], and consistently more than 25 out of the 27 MA delivered to the insulator stack reach the ZPDH load. The efficient convolute performance in $\mathrm{ZPDH}$ experiments is attributed to the relatively low initial load inductance and the moderate implosion velocity, which equates to a low rate of change of the load inductance, $d L / d t$. The voltage applied to the convolute can be approximated by

$$
V(t)=\left[L_{\text {load }}(t)+L_{\text {inner }}\right] \frac{d I(t)}{d t}+I(t) \frac{d L_{\text {load }}(t)}{d t},
$$

where $L_{\text {load }}$ is the time-dependent load inductance, $L_{\text {inner }}$ is the fixed inductance of the inner-MITL, and $I$ is the timedependent current. Assuming a perfectly conducting, coaxial, thin-shell representation for the load and return current path, the load inductance is given by

$$
L_{\text {load }}(t)=2 h * \ln \left[r_{0} / r(t)\right],
$$

where $L_{\text {load }}$ is the inductance in $\mathrm{nH}, h$ is the load height in $\mathrm{cm}, r_{0}$ is the radius of the outer conductor (return current path), and $r(t)$ is the radius of the load. The time derivative of the load inductance is given by

$$
\frac{d L_{\mathrm{load}}(t)}{d t}=0.002 h * \frac{v(t)}{r(t)},
$$

where $d L_{\text {load }} / d t$ is in units of $\mathrm{nH} / \mathrm{ns}, h$ is the load height in $\mathrm{cm}, v(t)$ is the radially inward velocity in $\mathrm{km} / \mathrm{s}$, and $r(t)$ is the load radius in $\mathrm{mm}$.

At early times, $I$ and $d L_{\mathrm{load}} / d t$ are small, and $d I / d t$ is large, so the first term of Eq. (1) dominates; near peak current, $d I / d t$ is small, and $I$ and $d L_{\text {load }} / d t$ are large, so the second term dominates. Thus voltage is minimized in loads with low initial inductance and low late-time implosion velocity. While these guidelines are useful for ensuring efficient delivery of current to the load, they are often in contradiction with experimental requirements, so it is useful to understand how the convolute performs under a variety of conditions. The initial inductance and approximate peak $d L / d t$ are compared to the peak convolute voltage and shunt current for a variety of experiment types in Table I.

As has been observed previously [18,20,38], the shunt current between the stack and the outer-MITL was negligible for all load types investigated. This is attributed to the relatively large AK gaps, which limited the impact of cathode plasma formation and motion in the outer MITLs. Also, by design, there is no anode plasma formation in the outer MITLs [40].

By contrast, large shunt currents were observed between the outer- and inner-MITL B-dot locations for some experiments. The convolute is located in between these current monitors, and the observed loss was attributed to it. The convolute shunt current ranged from approximately

TABLE I. A collection of the initial inner-MITL plus load inductance, approximate maximum implosion velocity, height of the imploding region, approximate peak rate of change of inductance, peak convolute voltage, and peak convolute shunt current for a variety of load types. The different objectives of these experiments required the wide range of initial $L$ and peak $d L / d t$, which impact convolute voltage and shunt current. Initial inductance was calculated assuming a perfectly conducting, coaxial, thin-shell approximation for the load and return current cylinder. This assumption underestimated the initial inductance by around $0.2-0.3 \mathrm{nH}$ in experiments with nonazimuthally continuous loads and/or return current cylinders (e.g., wire-array loads and slotted return current cylinders), which are marked with a \#. The values for $d L / d t$ were calculated at $r(t)=0.5 \mathrm{~mm}$, which is a good approximation for the radius at the time of peak implosion velocity in these experiments. Shunt current values in parentheses assume the 0.956x scale factor on the inner-MITL current described in Appendix A. Note that peak values for the convolute voltage and shunt current were not necessarily simultaneous.

\begin{tabular}{|c|c|c|c|c|c|c|}
\hline Experiment description & $L_{0}[\mathrm{nH}]$ & $v_{\max }[\mathrm{km} / \mathrm{s}]$ & $h[\mathrm{~cm}]$ & $d L / d t[\mathrm{nH} / \mathrm{ns}]$ & $V_{c}[\mathrm{MV}]$ & $I_{s}[\mathrm{MA}]$ \\
\hline $\mathrm{z2082}^{\#}-70-\mathrm{mm}$ SS wire array & 1.8 & 1000 & 2 & 8.0 & 2.9 & $7.3(8.3)$ \\
\hline z1992"-65-mm W wire array & 2.7 & 800 & 2 & 6.4 & 2.4 & $3.8(4.9)$ \\
\hline z2409 $^{\#}$-Z-pinch dynamic hohlraum & 2.7 & 300 & 1.2 & 1.4 & 1.7 & $1.8(3.2)$ \\
\hline z2172 -Short-pulse liner & 4.3 & 100 & 0.65 & 0.26 & 1.8 & $3.1(4.0)$ \\
\hline z2713 -Long-pulse liner, extended feed & 4.8 & 30 & 0.65 & 0.08 & 0.9 & $2.3(3.4)$ \\
\hline z2110 -Shaped-pulse liner & 4.9 & 50 & 0.65 & 0.13 & 1.7 & $2.4(3.5)$ \\
\hline z2708 ${ }^{\#-S h o r t-p u l s e ~ l i n e r, ~ e x t e n d e d ~ f e e d ~}$ & 7.0 & 70 & 1 & 0.28 & 2.6 & $9 *\left(10^{*}\right)$ \\
\hline
\end{tabular}
When marked with an *, the peak shunt current was estimated due to early B-dot failure, which was the result of an extremely high initial inductance. Traces from the experiments in bold are shown in Figs. 3-5. 

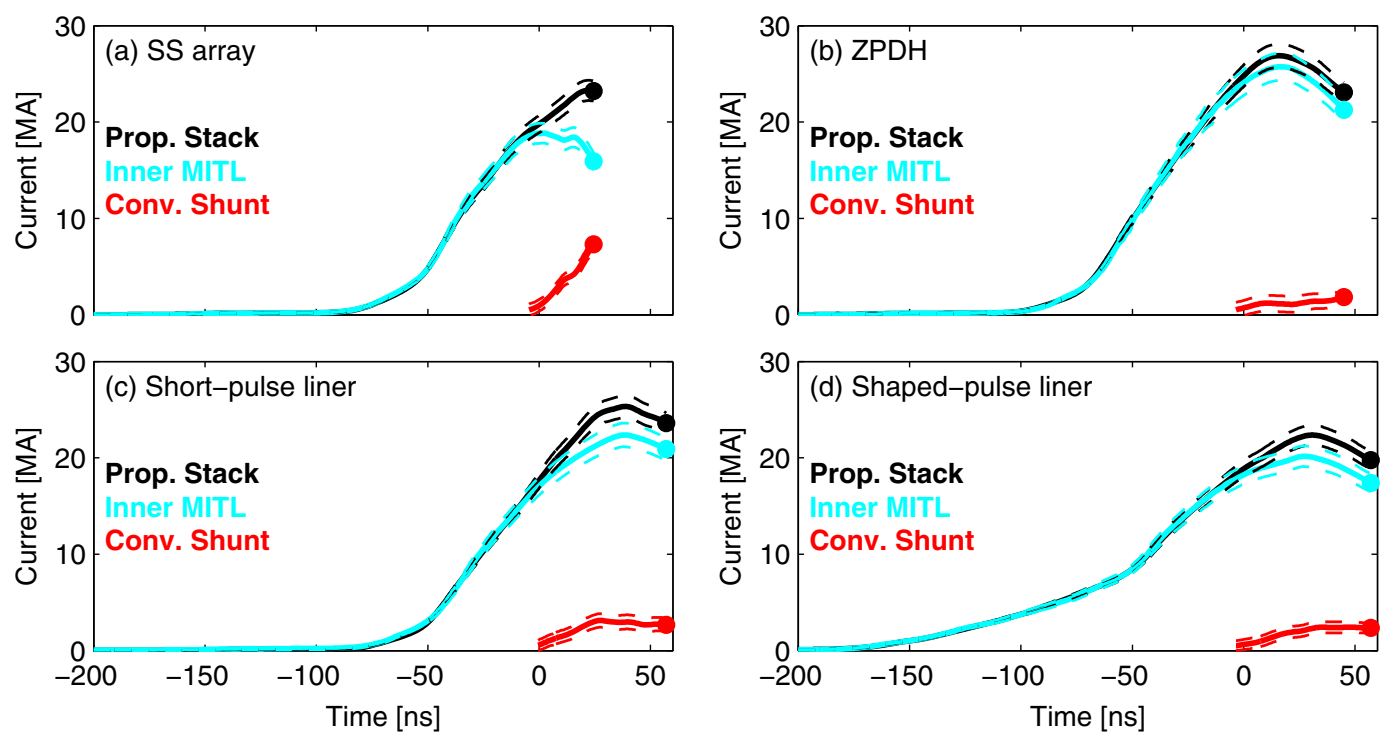

FIG. 3. (a)-(d) Plots of the losslessly propagated stack current (black), the inner-MITL current (cyan), and the difference between the two (red), called the convolute shunt current, for the four bolded load types from Table I. A dot at the end of each trace represents the time of stagnation. The time at which the convolute shunt impedance drops below $2 \mathrm{ohms}$ is defined as $t=0 \mathrm{~ns}$. Dashed lines indicate the uncertainty in each measurement [35].
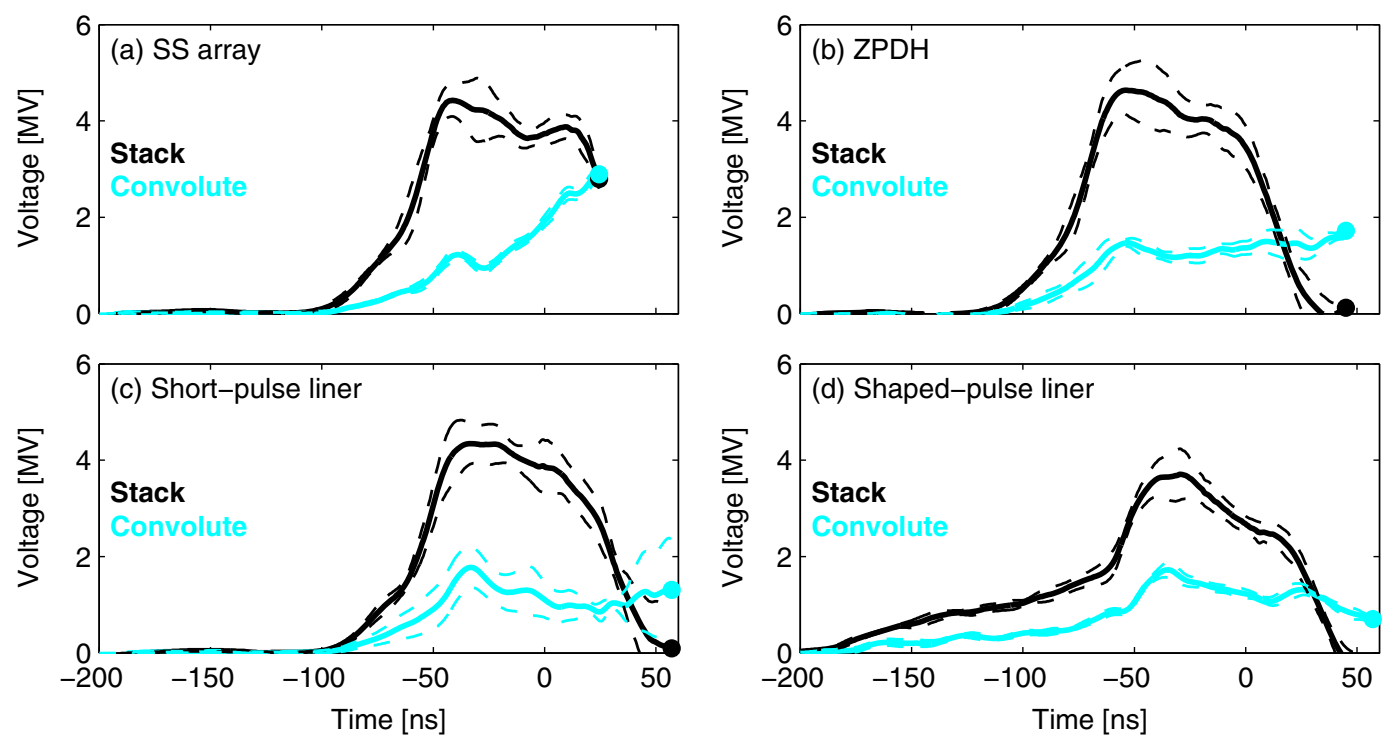

FIG. 4. (a)-(d) Plots of the stack voltage (black) and the convolute voltage (cyan) for the four bolded load types from Table I. The convolute voltage was determined using a lossless translation of the stack voltage [38]. A dot at the end of each trace represents the time of stagnation. The time at which the convolute shunt impedance drops below 2 ohms is defined as $t=0$ ns. Dashed lines indicate the uncertainty in each measurement [35].

1-2 MA (roughly the threshold of detection) in ZPDH experiments (low $L_{0}$, moderate $d L / d t$ ) up to 7-8 MA in experiments with wire arrays designed to produce intense K-shell x-ray emission [41] (low $L_{0}$, high $d L / d t$ ). Substantial convolute shunt currents were also inferred on ICF experiments in which an extended inner-MITL was required to accommodate applied-B-field coils [7,42] (high $L_{0}$, low $d L / d t$ ) but due to failure of the inner-MITL B-dots, a detailed analysis of the signals could not be performed. As noted above, the maximum recoverable drive current is about half the observed shunt current, even with lossless convolute performance.

Plots of the currents for the experiments listed in bold in Table I are shown in Fig. 3. In order to directly compare the various experiments, the traces were time shifted such that $t=0 \mathrm{~ns}$ occurred at the time that the convolute shunt 
impedance (details below) was equal to 2 ohms. The relative time shift between two experiments was typically less than $10 \mathrm{~ns}$, but in some experiments with a nonstandard current pulse shape it exceeded $20 \mathrm{~ns}$.

The uncertainty in the shunt current is a combination of the random uncertainties in the measured stack and inner-MITL currents and the systematic uncertainty in the B-dot measurements [35]. Typical uncertainties in the peak stack and inner-MITL currents are just over 1 MA (about 5\%).

Plots of the stack and convolute voltages for the same set of bolded experiments from Table I are shown in Fig. 4. The uncertainty in these measurements is given as the standard deviation of the average voltage of the four transmission lines [18] combined in quadrature with the systematic uncertainty of the D-dot measurements [35].

For short-pulse experiments, a local maximum was observed in the convolute voltage approximately $70 \mathrm{~ns}$ after the start of the signal; the local peak value was primarily determined by the initial inductance of the load and the charge voltage of the Marx banks. In low-initialinductance loads, this value was as low as $1 \mathrm{MV}$ and in high-initial-inductance loads it could approach $3 \mathrm{MV}$. In rapidly imploding loads $(>500 \mathrm{~km} / \mathrm{s})$ the convolute voltage continued to rise after the initial peak, and approached $3 \mathrm{MV}$ at stagnation. The voltage remained relatively constant at approximately $1.5 \mathrm{MV}$ in moderateimplosion-velocity $(300 \mathrm{~km} / \mathrm{s})$ experiments such as the ZPDH. In some slower implosion loads $(<100 \mathrm{~km} / \mathrm{s})$ the voltage decreased following the initial spike. Experiments with a shaped current pulse are also fielded on $Z$. The voltage in these experiments was applied for a much longer period (hundreds of ns), but it also was lower amplitude for the majority of the pulse.

The effective convolute shunt impedance was calculated by taking the ratio of the convolute voltage (Fig. 4) and shunt current (Fig. 3). This ratio was only meaningful when the shunt current was measurably nonzero. The uncertainty in the shunt impedance was calculated by combining the uncertainty in the convolute voltage and the convolute shunt current in quadrature. The shunt impedance had large uncertainty for small shunt currents, where the uncertainty in the shunt current was large. The uncertainty in the shunt impedance was very small at times with high loss. The convolute shunt impedances for nominally identical experiments was extremely repeatable, as shown in Fig. 5(a).

Despite large differences in shunt currents for different load types, the general form of the shunt impedance was relatively consistent across all load types. Initially no losses were observed, so the impedance was effectively infinite. At the time of collapse, the impedance dropped over a period of approximately $10 \mathrm{~ns}$ to a relatively constant value around $0.5-1 \mathrm{ohm}$. The shunt impedances for a variety of experiments are shown in Fig. 5(b). Note the impedance history for the ZPDH was considerably higher than that for other load
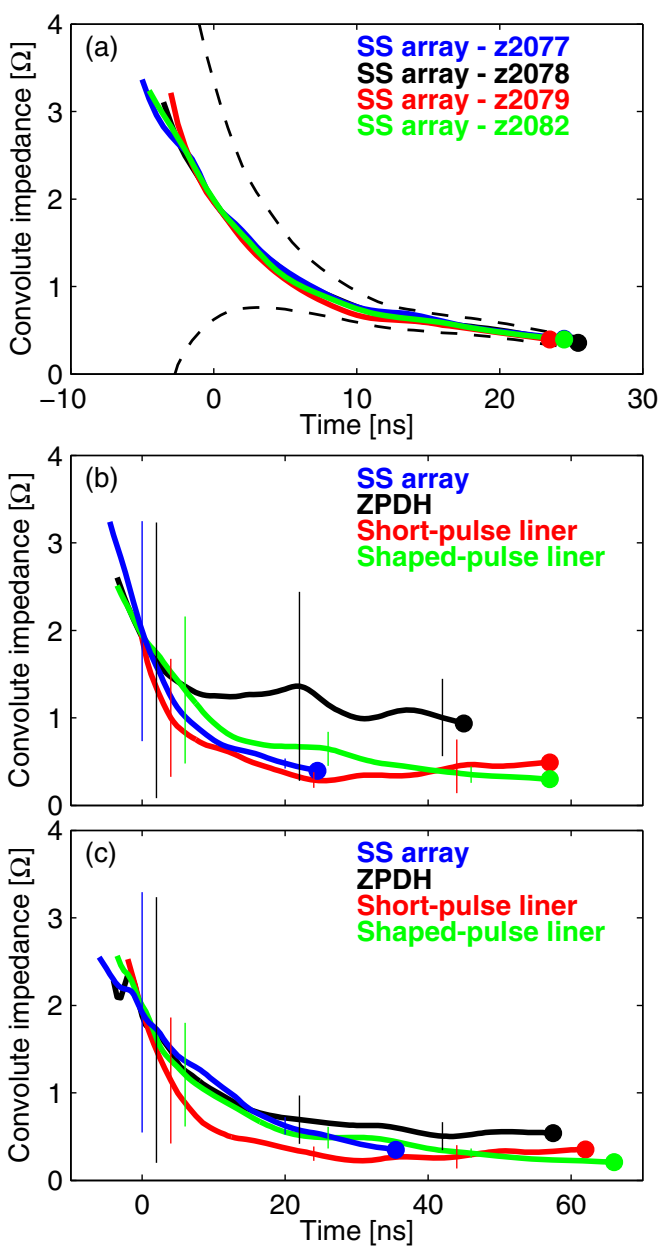

FIG. 5. (a) A plot of the impedance collapse in the convolute for four nominally identical 70-mm-diameter stainless-steel wirearray experiments. Dashed lines indicate the uncertainty in the impedance for one experiment (other uncertainties are omitted for clarity). For a given load type, the impedance history was typically repeatable to within the uncertainty in the measurement. (b) A plot of the impedance collapse in the convolute for the four bolded load types from Table I, assuming the standard B-dot calibrations. The general shape of the curves is similar; low-loss experiments appear to operate at slightly higher impedance, but the uncertainty in the measurement is much larger. A series of vertical lines indicates the uncertainty for each curve. (c) Same plot as (b) but assuming the systematic $0.956 \mathrm{x}$ correction to the inner-MITL B-dot calibrations detailed in Appendix A. Compared to (b), the impedance histories in (c) are more consistent with one another. A series of vertical lines indicates the uncertainty for each curve.

types, but the uncertainty in the measurement was relatively large due to the small convolute shunt current.

Experiments with a low-initial-inductance, nonimploding load have been conducted on the $Z$ machine to evaluate accelerator performance in the absence of losses. Details of the short-circuit experiments are given in Appendix A. In these experiments, the measured inner-MITL currents were systematically $4.6 \% \pm 2.1 \%$ higher than the losslessly 
translated stack current, which is nonphysical. To correct this, the inner-MITL currents could be decreased by $4.4 \%$ or the stack currents could be increased by $4.6 \%$. Given that the environment in which the inner-MITL B-dots resided was hostile (including $\mathrm{MeV}$ electrons and dense plasmas), the inner-MITL current measurements were less trusted, so the inner-MITL current was lowered rather than raising the stack current. Note that changing either current produces
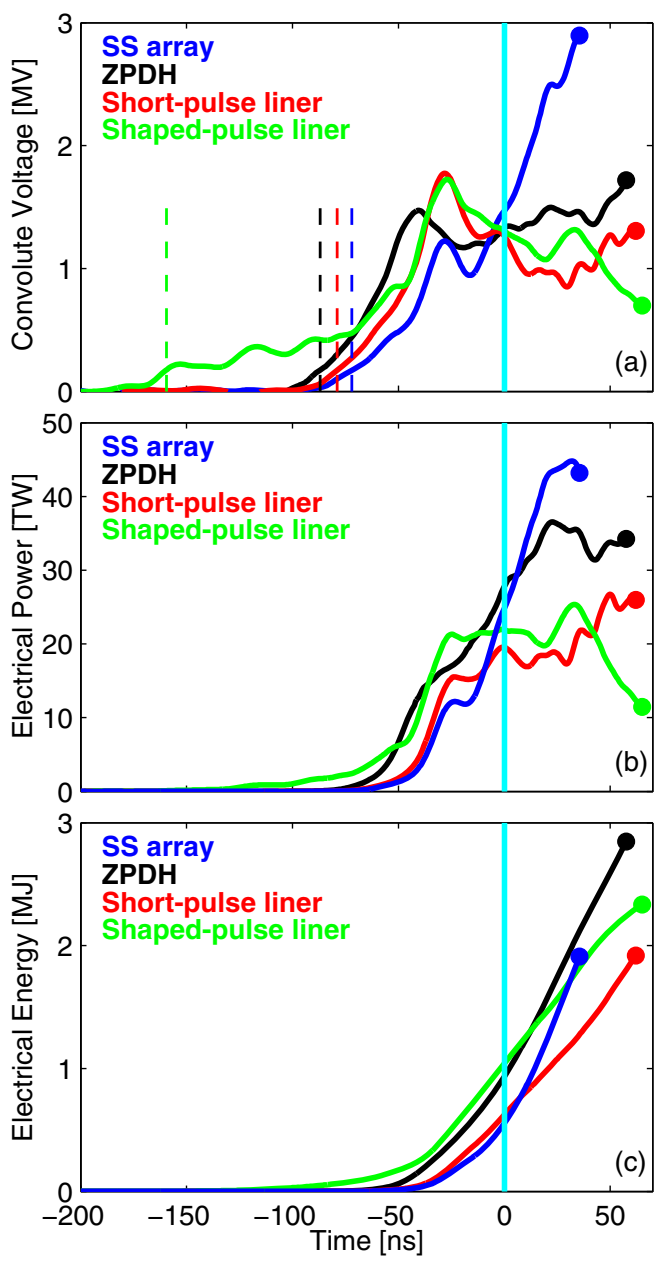

FIG. 6. (a) A plot of the convolute voltage for the four bolded load types from Table I. Dashed vertical lines indicate the time at which the electric field in the convolute exceeds the explosiveemission threshold of $240 \mathrm{kV} / \mathrm{cm}$. The solid vertical line at $t=0 \mathrm{~ns}$ indicates the time the shunt impedance drops below 2 ohms in each experiment. Coincidentally, all of the voltages passed through roughly the same value shortly before $t=0 \mathrm{~ns}$. Since some of the voltages had already exceeded this value and were decreasing, while others were rising through the value, it does not appear that there is any significance to this observation. There does not appear to be a simple correlation between applied voltage and impedance collapse. (b) A plot of the electrical power in the convolute for the same four experiments showing no obvious simple correlation. (c) A plot of the integrated energy passing through the convolute. Once again, there does not appear to be a simple correlation. similar convolute impedances curves. The convolute shunt currents and impedances were recalculated with the innerMITL current correction, and the impedances are plotted in Fig. 5(c). The $4.4 \%$ change in current is within the previously stated uncertainty in the inner-MITL current measurement. Because the shunt current in the $\mathrm{ZPDH}$ experiments was only approximately $3 \%$ of the total current, the change to the inner-MITL current calibration had the largest impact on the ZPDH shunt impedance. This brought the ZPDH impedance much closer to the impedances observed in the other three experiment types. The convolute impedance for these experiments is reasonably well fit by

$$
Z(t)=1.5 e^{-0.082 t}+0.31
$$

where $Z$ is the convolute shunt impedance in ohms and $t$ is the time in ns relative to the time when the impedance drops below 2 ohms.

While the shunt impedance was fairly consistent from one experiment to the next, the timing of the impedance decay is not well predicted with simple models. Recall that various experiments required relative time shifts of up to 21 ns to align the times of impedance collapse. Figure 6(a) shows the collapse time does not appear to be directly correlated to the time the electric field exceeds the explosive-emission threshold of $240 \mathrm{kV} / \mathrm{cm}$ (cathodeplasma formation). Additionally, it is not directly correlated to the electrical power reaching the convolute [Fig. 6(b)] or the total electrical energy delivered to the convolute [Fig. 6(c)]. An advanced version of the $Z$ circuit model [40], which is designed to predict the timing of the impedance collapse, is under development [43].

\section{SPECTRAL MEASUREMENTS}

Visible emission from the convolute region was collected and transported via fiber optic to two streaked-visiblespectroscopy (SVS) diagnostics. Similar systems have been used at Sandia to understand applied-B ion diodes [44,45] and shocks in liquid deuterium [46]. Details of the SVS diagnostics are given in Appendix B.

The SVS probe used in the convolute measurements was inserted into a vacant inner-MITL B-dot position and was designed to look electrically similar to a B-dot probe [47]. The SVS probe had a roughly axial view through the convolute, with the line of sight passing through the holes in the upper and lower cathodes just downstream of the convolute posts, as shown in Fig. 7(a). The angle of the probe could be set to 0,5 , or 7.5 degrees relative to vertical, which allowed for measurements near the cathode, in the middle of the upper-post-hole gap, and near the anode post. The fiber was aperture limited with a full-angle view of 4.1 degrees. This reduced the collection efficiency by a factor of 40 compared to a bare fiber, but allowed for coarse spatial resolution. In the upper post-hole region, the probe 

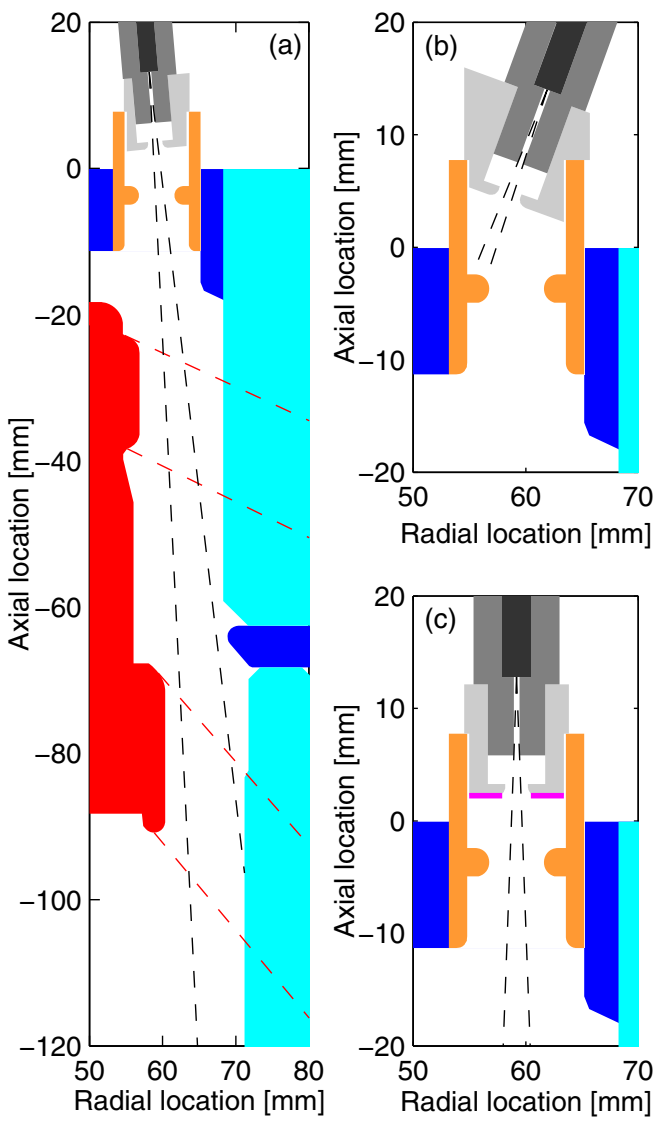

FIG. 7. (a) An $R-Z$ cross section of the convolute showing the field of view of the convolute-spectroscopy probe. The probe observed visible emission from the region downstream of the convolute post, where simulations indicated that plasma would collect. Dashed black lines indicate the field of view of the probe. The angle of the probe could be set to 0,5 , or 7.5 degrees relative to vertical (5 degrees shown). (b) An $R-Z$ cross section of the probe view in a null experiment. The probe could only see the inside of the aperture in the anode. No signal was observed in these measurements indicating minimal plasma formation at the probe. (c) An $R-Z$ cross section of a convolute-spectroscopy probe with a lithium fluoride $(\mathrm{LiF})$ dopant on the bottom surface of the probe, shown in magenta. No lithium features were observed in this measurement, again indicating minimal plasma formation at the probe.

collected signal from a conical section with diameter increasing from 2.8 to $4.0 \mathrm{~mm}$ [intersections of red and black dashed lines in Fig. 7(a)].

Within the vacuum chamber, the fibers were shielded with a flexible $0.5 \mathrm{~mm}$ thick copper braid. This dramatically reduced the sub-30 keV x-ray fluence incident on the fiber, which essentially eliminated the background caused by $x$-ray interactions in the fiber.

Survey measurements of the convolute emission were conducted on several experiments to cover the entire visible spectrum (4000-7000 $\AA$ ). The spectra consisted of continuum emission with a few absorption lines. No clear emission lines were observed. The fact that spectral lines were only observed in absorption indicates that the source of the continuum emission was substantially brighter (hotter and/or denser) than the source of the spectral lines. In all of these measurements, a portion of the probe line of sight included an electrode surface, which was likely the source of the bright continuum.

The most prominent absorption line was the neutral hydrogen line at $6563 \AA(\mathrm{H} \alpha)$; the neutral hydrogen line at
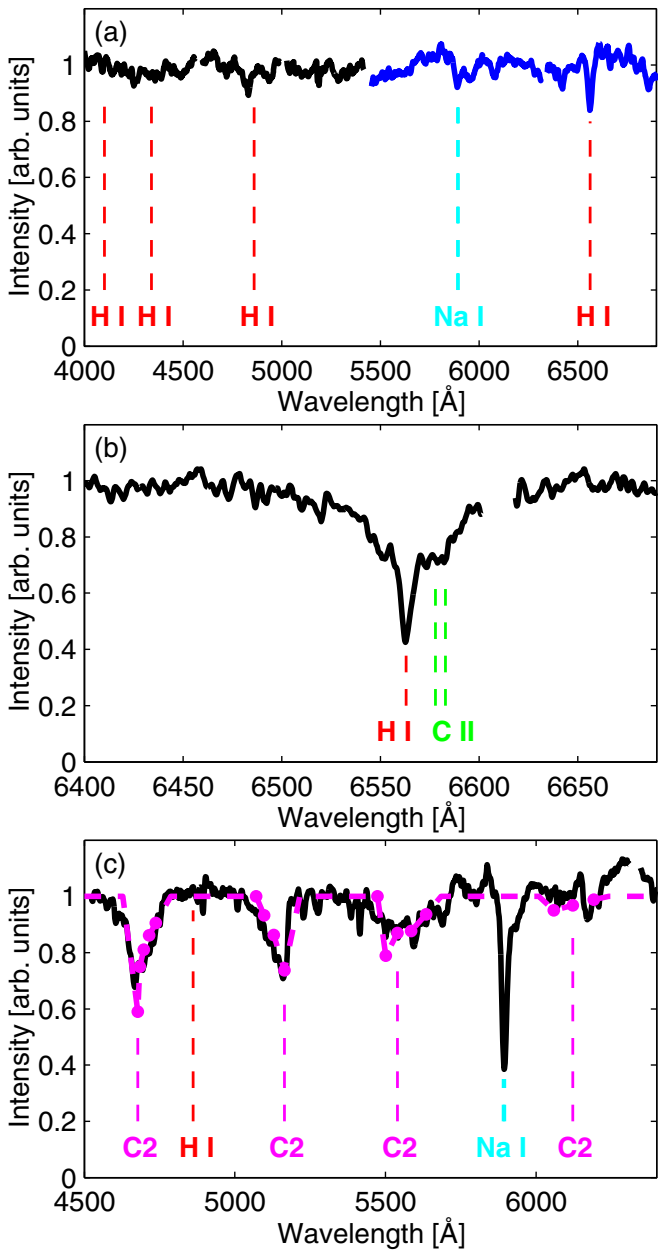

FIG. 8. (a) Visible spectra from two experiments near stagnation are shown in black $(t=31 \mathrm{~ns})$ and blue $(t=24 \mathrm{~ns})$. Breaks in the signals at $4579,5050,5435$, and $6328 \AA$ are due to spectral and temporal calibration features. Vertical lines indicate the expected location of hydrogen and sodium spectral lines. There was a clear neutral hydrogen (H I) feature at $6563 \AA$ and possible features associated with the neutral sodium ( $\mathrm{Na}$ I) doublet at $5890 / 5896 \AA$ A. There are no obvious spectral features at 4861 , 4340 , or $4102 \AA$. There are possible spectral features at 4831 and $4937 \AA$, which have not yet been identified. (b) A post-stagnation ( $t=94 \mathrm{~ns})$, high-spectral-resolution plot of the H I (6563 $\AA$ ) and singly ionized carbon (C II) lines at 6578 and $6583 \AA$. (c) A plot of a post-stagnation spectrum $(t=88 \mathrm{~ns})$ showing $\mathrm{C}_{2}$ absorption features (Swan bands) as well as the $\mathrm{Na}$ I doublet at $5890 / 5896 \AA$ A. The magenta dots represent the most dominant spectral bands of the Swan system to aid in spectral identification (modified from Table I in Ref. [48]). 
$4861 \AA(\mathrm{H} \beta)$ was not clearly observed. Due to its prominence, the $\mathrm{H} \alpha$ line was the focus of additional high-spectral-resolution measurements. Other spectral features observed in absorption included the neutral sodium (Na I) doublet at 5890 and $5896 \AA$, singly ionized carbon (C II) lines at 6578 and $6583 \AA$, and, at late times, $\mathrm{C}_{2}$ molecular features [48] at roughly 4640-4760 $\AA$, 5080$5180 \AA$ and $5460-5700 \AA$. Example spectra are shown in Fig. 8. These spectroscopic measurements were used to determine the composition, location, density, temperature, apparent closure velocity, and radial velocity of the plasma.

An underlying assumption in the following analysis is that the neutral hydrogen absorption line is an indicator of the presence of dense plasma, which is partially ionized. The signature of neutral hydrogen is used to infer conditions of the bulk plasma. This assumption should be valid for the inferred temperatures (few $\mathrm{eV}$ ) and densities $\left(1 \times 10^{16}-1 \times 10^{18} \mathrm{~cm}^{-3}\right)$.

\section{A. Composition and location}

Due to the high electrical power density $\left(\sim \mathrm{TW} / \mathrm{cm}^{2}\right)$ in the convolute, plasma formation on the probe was a concern [49]. A series of experiments were conducted to confirm that the observed spectral features originated in the convolute, as opposed to on the probe itself. Several null tests were conducted in which the probe could only collect emission from within the B-dot recess in the anode, as shown in Fig. 7(b). No signal was observed in these experiments, which indicates that any plasma formation within the recess and/or on the probe itself did not produce the signals observed in other measurements with similar probe designs.

An additional null test with a lithium fluoride $(\mathrm{LiF})$ dopant, as depicted in Fig. 7(c), was conducted. Lithium was chosen because it was not observed in any previous spectra, so if observed, the source of the signal could be isolated. Additionally, there is a strong neutral lithium line at $6708 \AA$, which has been observed previously in highpower diodes $[44,45]$. In this experiment, the bottom of the SVS probe was coated with $1 \mu \mathrm{m}$ of LiF. The probe had a field of view similar to the one shown in Fig. 7(a). The recorded spectrum consisted of continuum emission and the $\mathrm{H} \alpha$ absorption line, but no lithium features were observed. This further demonstrated that there was not significant plasma formation on the probe.

A measurement was conducted using a LiF dopant to positively confirm that the observed signals originated in the convolute [47]. A $1 \mu \mathrm{m}$ thick, $1 \mathrm{~mm}$ tall band of LiF was applied to the upper convolute post as shown in Fig. 9. Emission collected on this experiment included an absorption feature from the neutral lithium (Li I) line at $6708 \AA$. Because this spectral feature was backlit by the continuum emission, at least a portion of the source of the continuum must have been located below the lithium. The only possible source of emission below the lithium was plasma
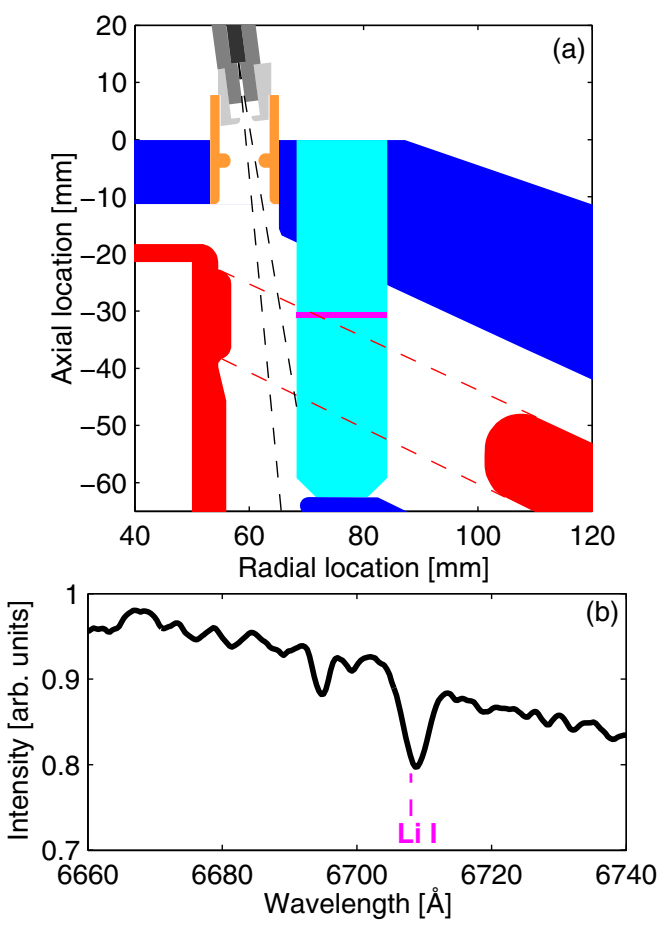

FIG. 9. (a) An $R-Z$ cross section of the convolutespectroscopy-probe field of view (black dashed lines) with a lithium fluoride (LiF) dopant (magenta band) located on the upper convolute post (anode). (b) A plot of the spectrum showing the neutral lithium (Li I) absorption line at $6708 \AA$. This demonstrated that a continuum source was located below the position of the Li dopant. The spectrum shown was averaged over $t=24$ to $104 \mathrm{~ns}$ in order to obtain a high signal to noise ratio. The Li I spectral feature was first clearly observed at $t=24 \mathrm{~ns}$.

within the convolute (either at an electrode surface or within the anode-cathode gap). The observation of lithium neutrals from the anode indicated that anode plasmas were being evolved from processes in the convolute.

Despite the fact that the electrodes in the region were stainless steel (coated with $3 \pm 0.5 \mu \mathrm{m}$ of gold in some experiments), no $\mathrm{Fe}, \mathrm{Cr}, \mathrm{Ni}, \mathrm{Mn}, \mathrm{Mo}$, or Au lines could be identified. All identified lines were the result of contaminants, thus the initial inventory of plasma was probably dominated by those contaminants. Hydrogen and carbon likely formed from desorption, fragmentation, and ionization of water and various hydrocarbons adsorbed on the electrode surfaces [50,51]. At the typical vacuum base pressures of these experiments $\left(5 \times 10^{-5}\right.$ Torr $), \mathrm{H}_{2} \mathrm{O}$ was $80 \%-90 \%$ of the partial pressure of the vacuum background [52], so presumably it dominated the most loosely bound and first-desorbed electrode adsorbates. The sodium is attributed to unintended direct human-electrode contact. Negligibly small quantities (nanograms) have been readily observed in visible spectra from other experiments on $Z$ [53]. The dominance of contaminants in the convolute plasma inventory is consistent with spectroscopic observations in previous pulsed-power systems (electron-beam 
diodes, ion-beam diodes, MITLs) [54]. Desorption rates of surface contaminants are orders of magnitude greater than the vapor pressures of electrode materials, until electrodes reach melt and vaporization [54].

Continuum emission was observed in the convolute once the current exceeded approximately $10 \mathrm{MA}$, as shown in Fig. 10(a). For otherwise nominally identical experiments, a measurement with a near-cathode view (0-degree probe) observed both the continuum emission and the $\mathrm{H}$ and $\mathrm{C}$ lines earlier than in an equivalent measurement with a gap view [5-degree probe; view shown in Fig. 7(a)]. The $\mathrm{H} \alpha$ line was typically observed within $10 \mathrm{~ns}$ of the start of continuum emission for both views, and the $\mathrm{C}$ and $\mathrm{Na}$ lines were not typically observed until around or after stagnation (approximately 30-35 ns later). These observations indicate that the hydrogen expanded into the gap at a higher velocity than the other contaminant species, and the main source of the hydrocarbons was likely the cathode. The assumption of a pure hydrogen plasma used in most simulations appears reasonably valid for the majority of the duration of the experiment; however, the assumption of a completely ionized plasma in most simulations should be revisited.

The molecular emission lines of $\mathrm{C}_{2}$ observed late in time in the convolute were also previously observed late in the
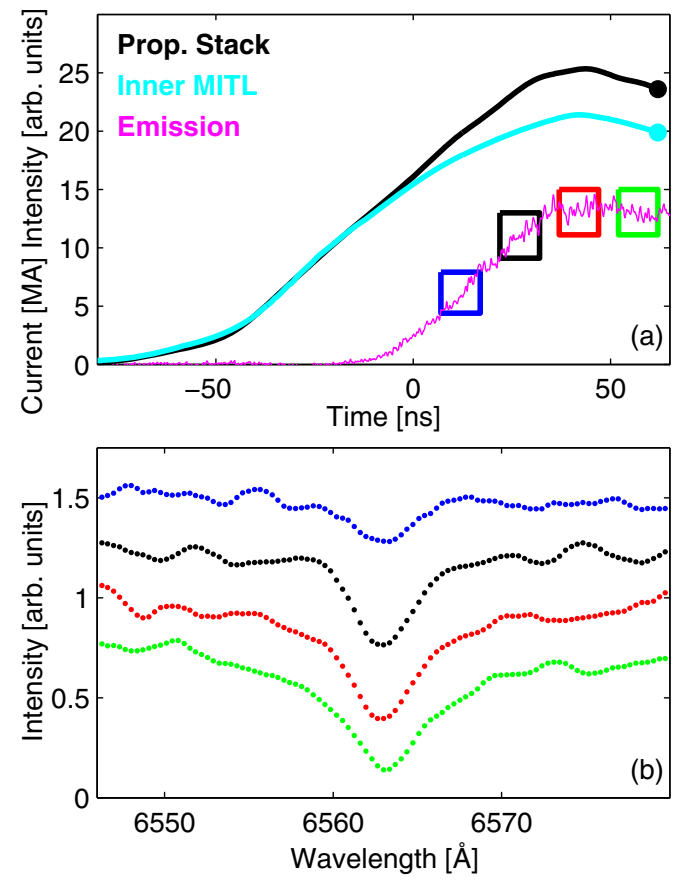

FIG. 10. (a) A plot of the propagated stack and inner-MITL currents with the convolute plasma emission intensity in the 6400 to $6450 \AA$ spectral band as a function of time. Boxes over the emission signal indicate the times over which the spectral lineouts in (b) were taken. (b) Spectra showing the evolution of the neutral hydrogen feature at $6563 \AA$ with time. The spectra have been normalized to unity and then offset by $+0.5,+0.25,0$, and -0.25 for clarity. electrical pulse in electron-beam-diode experiments [55]. A subset of these molecular features is known as the Swan bands, which are characteristic of burning hydrocarbon fuels and result from underlying chemical kinetics of combusting hydrocarbons [48]. In pulsed-power systems, such emission may be related to expanding and cooling electrode plasmas formed from hydrocarbon contaminant constituents.

\section{B. Density and temperature}

Simple models of the convolute plasma were generated in PrismSPECT [56]. Details of the simulation setups and the generated spectra are given in Appendix C. Based on a comparison of the simulated and experimentally observed lines, the temperature in regions of the plasma could have spanned from less than $1 \mathrm{eV}$ to nearly $4 \mathrm{eV}$. The temperature range where all of the spectral features could have been observed simultaneously was $2-2.5 \mathrm{eV}$. This is consistent with previous electrode-plasma temperature estimates at these linear current densities $(0.1-1 \mathrm{MA} / \mathrm{cm})$ [57-59].

High-resolution measurements centered at $6560 \AA$ were used to monitor the evolution of the $\mathrm{H} \alpha$ and $\mathrm{C} \mathrm{II}$ absorption features on a variety of experiments. As shown in Fig. 10, the $\mathrm{H} \alpha$ feature broadened rapidly at late times. The $\mathrm{H} \alpha$ line shape was used to infer the electron density as a function of time. Assuming the ion and electron temperatures were equal, the tables in Ref. [60] were used to relate line shape to electron density.

Spectral lineouts were averaged over a 10-ns window, and the continuum was flattened and normalized to unity. The $\mathrm{H} \alpha$ absorption was fit with a superposition of up to two

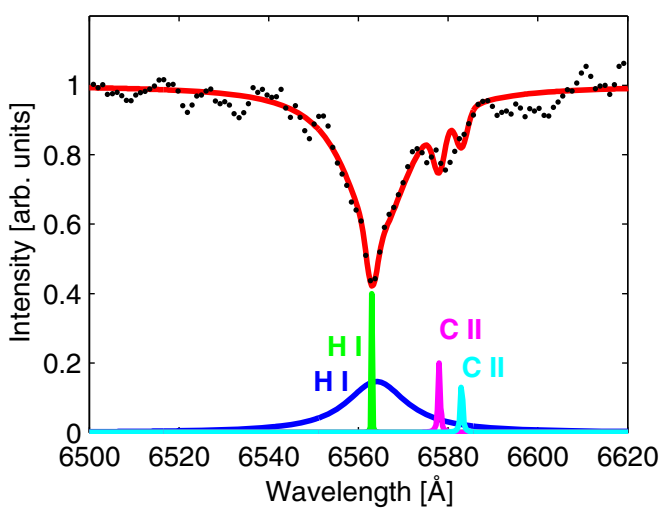

FIG. 11. A plot of the high-resolution spectrum shortly after stagnation ( $t=67 \mathrm{~ns}$ ) showing continuum emission with neutral hydrogen (H I) and singly ionized carbon (C II) absorption features (black dots). A fit to the data is shown in red. The fit is a superposition of two Lorentzian profiles for the H I line at $6563 \AA$ and two additional Lorentzian profiles for C II lines at 6578 and $6583 \AA$, which is convolved with a $2.7 \AA$ FWHM Gaussian profile to account for instrumental broadening. The individual Lorentzians used in the fit are shown below. 
Lorentzian profiles convolved with a Gaussian profile, which accounted for the instrumental broadening (typically $2.7 \AA$ FWHM). At early times, a single Lorentzian profile was sufficient to fit the data, but at late times, $\mathrm{H} \alpha$ was better fit by two profiles: one with an equivalent density of about $1 \times 10^{16} \mathrm{~cm}^{-3}$ and the other between $1 \times 10^{17}$ and $1 \times 10^{18} \mathrm{~cm}^{-3}$. This dual density fit to $\mathrm{H} \alpha$ is not a unique solution, but it demonstrates that the plasma likely had a density gradient that covered the range of densities used in the fit. In addition to $\mathrm{H} \alpha$, a pair of nearby $\mathrm{C}$ II absorption lines were included in the fit. An example spectrum with the fit is shown in Fig. 11. Given the typical resolution of the instrument, the SVS measurements were only sensitive to densities in excess of $1 \times 10^{16} \mathrm{~cm}^{-3}$.

The uncertainty in the inferred density is a combination in quadrature of several sources of uncertainty. The uncertainty in the FWHM of the fit, which ranged from sub- $\AA$ at low densities to approximately $\pm 10 \AA$ at high densities, corresponds to approximately a $25 \%$ uncertainty in the density (on average), with larger uncertainty at lower densities. An additional uncertainty was introduced by assuming the plasma temperature was $2 \mathrm{eV}$. Given a $\pm 1 \mathrm{eV}$ uncertainty in temperature, the resulting additional uncertainty in the density was approximately 13\% [60].

\section{Correlation of impedance and density}

Sufficiently dense plasma in contact with an electrode will act as an extension of that electrode, effectively reducing the A-K gap. Plasma with density below the detection threshold of the SVS diagnostic $\left(\sim 1 \times 10^{14} \mathrm{~cm}^{-3}\right)$ is capable of spacecharge-limited electron emission [22,61]. Since the spectral measurements are sensitive to plasma densities in excess of $1 \times 10^{16} \mathrm{~cm}^{-3}$, they could not give the exact time of plasma electrode formation; they could only demonstrate that a plasma electrode formed prior to the observed density rise. A comparison of the time history of the density to the electrical signals (Fig. 12) shows that the density rose rapidly after the convolute shunt impedance collapsed.
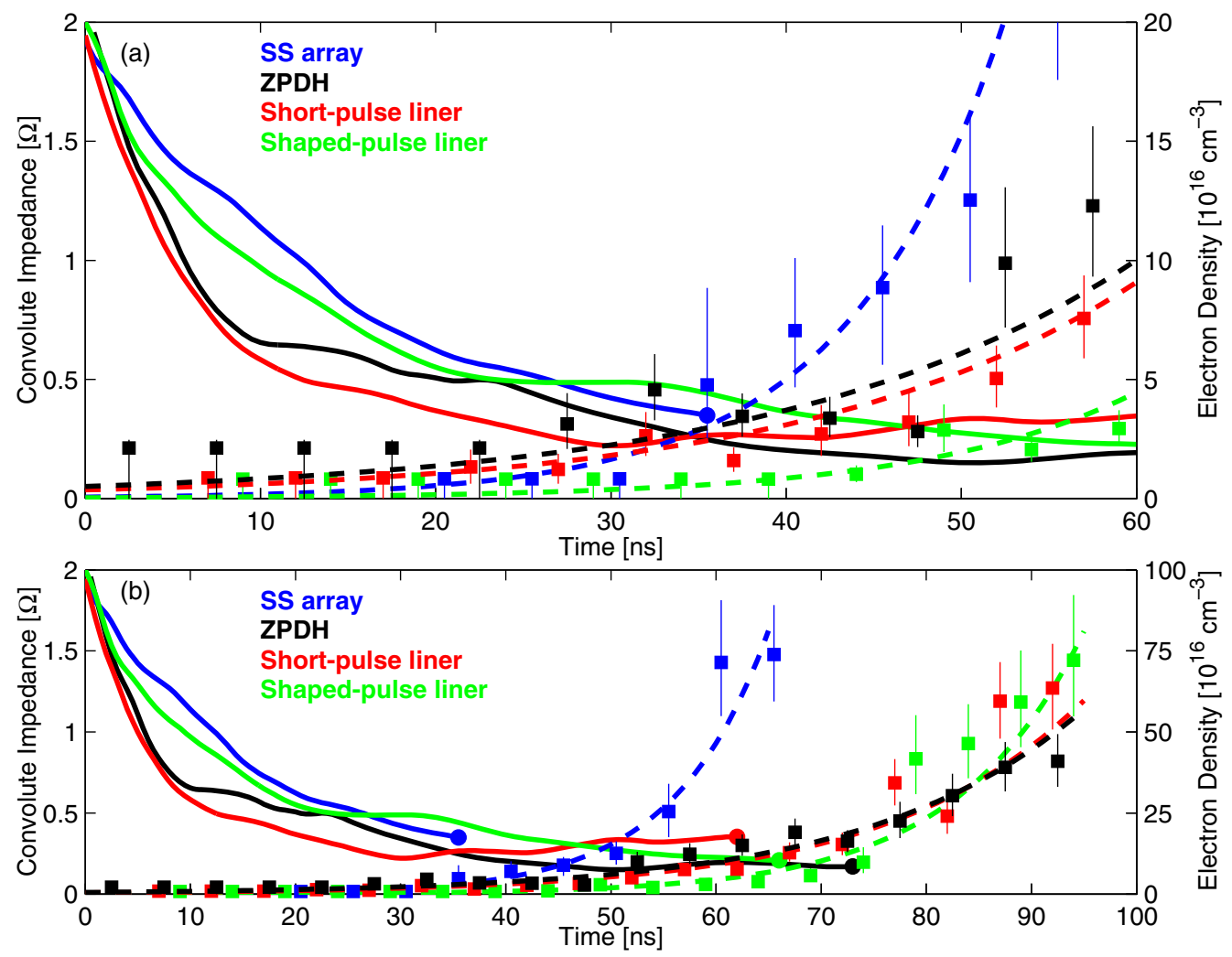

FIG. 12. (a) Plot of the convolute impedance (solid lines) and plasma electron density (squares) for a variety of load types. The dashed lines are exponential fits to the inferred densities. Vertical lines indicate the uncertainty in the density measurement. At early times the width of the absorption feature was dominated by instrumental broadening, which typically limited the measurement to densities above $1 \times 10^{16} \mathrm{~cm}^{-3}$. Note that the SVS was slightly out of focus on the ZPDH experiment resulting in a measurement threshold of approximately $2 \times 10^{16} \mathrm{~cm}^{-3}$. In all experiments, the electron density rose to a detectable level as impedance dropped, and typically exceeded $1 \times 10^{17} \mathrm{~cm}^{-3}$ at or shortly after stagnation of the z-pinch load. In early-time measurements, where the spectral line shape was dominated by instrument broadening, the densities are represented by points at the measurement threshold. (b) The same plot shown in (a) including later times and higher densities. Note when a superposition of two Lorentzians was fit to the data, the larger FWHM was used to infer the density. 
This implies that the plasma density in this region exceeded $1 \times 10^{14} \mathrm{~cm}^{-3}$ (allowing a plasma electrode to form) at an earlier time, but it is not clear exactly when it occurred relative to the start of the impedance collapse.

Despite the substantially different voltage histories applied to the convolute and the large difference in shunt currents for the different types of $z$-pinch loads, the electrondensity histories for these experiments followed a similar trend. In each case, the density was typically less than $1 \times 10^{16} \mathrm{~cm}^{-3}$ for the majority of the experiment; after the start of the impedance collapse, the density rapidly increased and normally reached $1 \times 10^{17} \mathrm{~cm}^{-3}$ by stagnation. In most experiments, the density peaked around $1 \times 10^{18} \mathrm{~cm}^{-3}$ well after stagnation. The density curves for the $\mathrm{ZPDH}$, shortpulse liner, and shaped-pulse liner experiments were similar to one another, which reinforces the hypothesis that the convolute behavior was similar across a variety of experiments, and the observed losses were primarily driven by the voltage applied to the convolute. In general, there appears to be a trend towards a slightly later density rise in experiments with higher impedance, although the differences in impedance were generally within the uncertainty in the measurements of one another.

While the general behavior of plasma electron density was similar across these experiments, there was one relatively dramatic difference. For the SS wire array, which produced nearly $3 \mathrm{MV}$ across the convolute at stagnation (compared to 1-2 MV for the other experiments), the density rose more quickly post-stagnation, rapidly approaching $1 \times 10^{18} \mathrm{~cm}^{-3}$ approximately $30 \mathrm{~ns}$ prior to the other experiments, as shown in Fig. 12(b). This may be a sign that although the convolute impedance collapse looked very similar for the different experiments, the plasma dynamics could diverge from nominal for extremely high-stress experiments at late times. However, it is worth noting that the time at which the inferred density approached $1 \times 10^{18} \mathrm{~cm}^{-3}$ relative to the stagnation time in each experiment was similar, so the latetime spike in density may be related to post-stagnation effects rather than the convolute impedance collapse. For example, an extremely intense burst of $\mathrm{x}$ rays bathes the inner-MITL and convolute at stagnation, which could enhance plasma formation and motion in the region. This effect could be more pronounced in the wire-array implosions, since they can generate hundreds of TW of $\mathrm{x}$ rays [41].

It is worth reemphasizing that due to diagnostic limitations, much of the interesting physics occurred prior to the time at which the plasma conditions were diagnosed with these spectral measurements. The time at which the plasma density exceeded $1 \times 10^{17} \mathrm{~cm}^{-3}$ may not be directly linked to the time at which the density reaches $1 \times 10^{14} \mathrm{~cm}^{-3}$ and a plasma extension of the cathode forms. Note that there is precedent for using latetime behavior to understand related, but undiagnosable, early-time effects [62]; however, any conclusions drawn from comparing late-time plasma densities are softened by the assumption that the density rate of rise between $1 \times 10^{16}$ and $1 \times 10^{18} \mathrm{~cm}^{-3}$ is directly correlated to the density rate of rise around $1 \times 10^{14} \mathrm{~cm}^{-3}$.

\section{Post to post symmetry}

Typically, convolute simulations have consisted of a 30degree azimuthal section of the geometry centered about the post with reflective boundary conditions in between posts or a 15-degree azimuthal section with reflective boundaries at the center of the post and between posts [11,22-28]. Both cases assume that the plasma formation and losses are consistent from one post hole to the next. The plasma density histories for identical views at two different posts were compared to test this assumption on a pair of experiments. The inferred plasma densities at both posts rose at a similar rate and reached a similar peak, as shown in Fig. 13. The difference in timing between the exponential fits to the two data sets was less than $3 \mathrm{~ns}$ for the range of $2 \times 10^{17}$ to $4 \times 10^{17} \mathrm{~cm}^{-3}$. Assuming this consistency existed at early times with low densities, this indicates that the 12-fold azimuthal symmetry used in convolute simulations is reasonable, although a slight, random staggering of the timing between posts may be appropriate in future simulations to account for the up to 3 ns variation in timing between the exponential fits to the data.

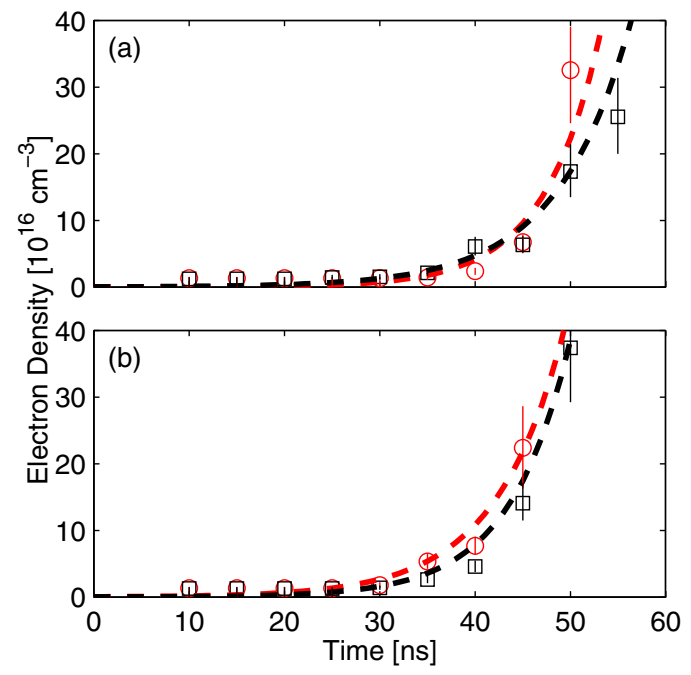

FIG. 13. (a) A plot of the electron density at two different azimuthal locations (red circles and black squares) and exponential fits to the data for a shaped-pulse liner experiment. (b) A plot of the electron density at two different azimuthal locations (red circles and black squares) and exponential fits to the data for a different shaped-pulse liner experiment. The timing uncertainty between the two spectroscopy systems was approximately $1 \mathrm{~ns}$, and each spectrum was integrated over $\pm 5 \mathrm{~ns}$ from the plotted point. These data sets indicate that the plasma density increased at a similar rate at each convolute post. 


\section{E. Apparent expansion velocity}

The density inferences mentioned above required neutral hydrogen in the line of sight, which was often located at least $3 \mathrm{~mm}$ from the nearest electrode surface. A typical expansion velocity for a $2 \mathrm{eV}$ hydrogen plasma is $2 \mathrm{~cm} / \mu \mathrm{s}$ [63], but at this rate the hydrogen would not enter the line of sight for $150 \mathrm{~ns}$. The earliest measurements of hydrogen absorption occurred approximately $70 \mathrm{~ns}$ after the start of current, which would require an expansion velocity of at least $4 \mathrm{~cm} / \mu \mathrm{s}$. Similarly, the Li dopant from the experiment described in Fig. 9 was observed $4 \mathrm{~mm}$ from the anode post approximately $100 \mathrm{~ns}$ after the start of current, implying at least a $4 \mathrm{~cm} / \mu \mathrm{s}$ velocity. These observations indicate that neutral particle expansion from both the anode and cathode occurred at more rapid rates than typical dense plasma expansion rates observed in MITLs.

In a previous study, the turn-on times of the continuum emission at a few radial positions in the upper post-hole region were used to infer that the convolute plasma formed at the cathode and progressed towards the post (anode) [47]. This measurement relied on several nominally identical Z-pinch experiments, each with a different SVS-probe alignment angle. Based on these measurements, the closure velocity was estimated to be $>7 \mathrm{~cm} / \mu$ s [47]. Concerns over the location of the continuum emission source, the angle of the observation line of sight, and the shot-to-shot reproducibility of the emission prompted this measurement to be revisited.

A probe was designed to allow multiple measurements in the same post hole on the same experiment, as shown in Fig. 14, which allowed coarse spatial resolution of the

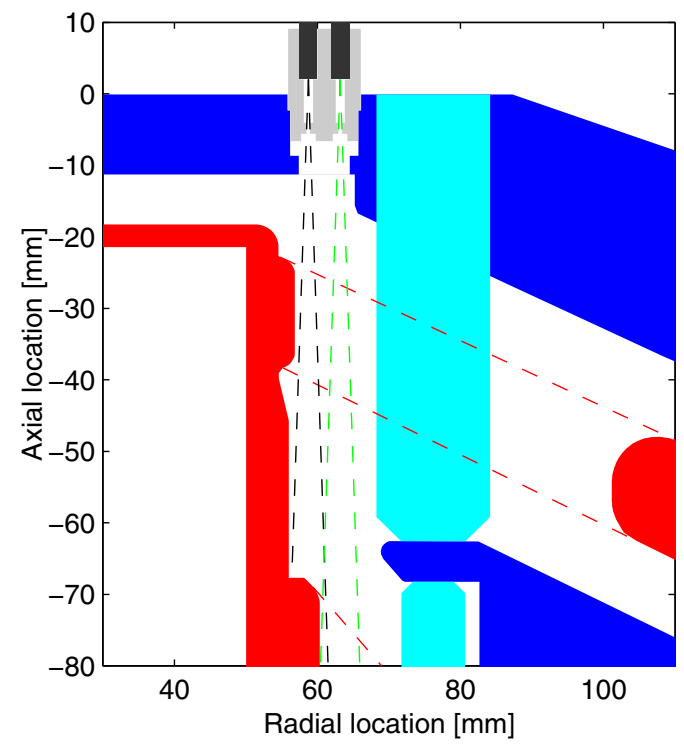

FIG. 14. An $R-Z$ cross section showing the field of view of the two lines of sight in the dual-view probe. The cathode view is shown in black dashed lines and the anode view is shown in green dashed lines. The separation of the center of the two lines of sight is $4.5 \mathrm{~mm}$. inferred electron density versus time. Measurements of the $\mathrm{H} \alpha$ absorption feature were used to unfold the density history at two locations on several experiments. In each experiment, the density rapidly rose to greater than $1 \times 10^{17} \mathrm{~cm}^{-3}$ in the line of sight closer to the cathode first, and the density rose in the line of sight closer to the post approximately 9-13 ns later, as shown in Fig. 15. This supports the hypothesis that plasma responsible for the $\mathrm{H} \alpha$ line broadening was expanding from the cathode towards the anode.
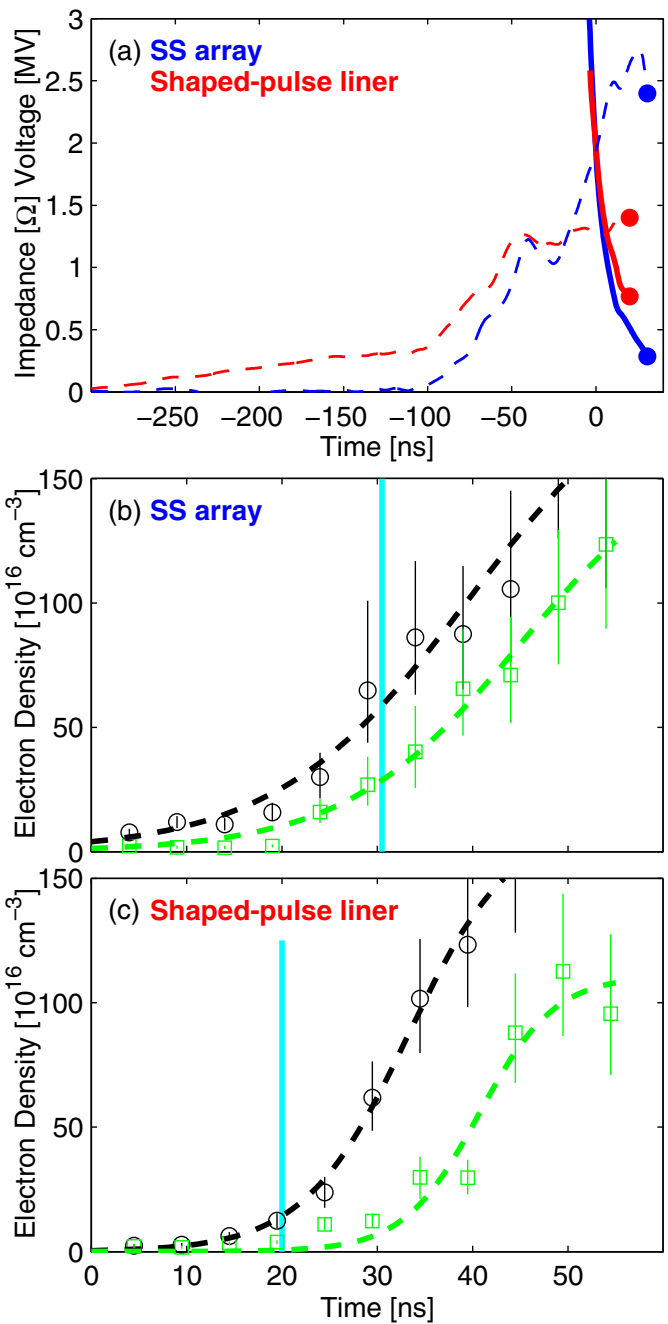

FIG. 15. (a) A plot of the convolute voltage (thin dashed) and impedance (thick solid) as a function of time for two different load configurations. (b)-(c) Plots of the electron density from the cathode view (black circles) and anode view (green squares) for the two experiments with Sigmoid curves fit to the data sets. The solid, vertical, cyan line indicates the time of stagnation. The delay between the fits to the cathode and anode view data sets was around 9-10 ns for the SS array and around 11-13 ns for the shaped-pulse liner for densities between $2 \times 10^{17}$ and $4 \times 10^{17} \mathrm{~cm}^{-3}$. These delays correspond to closure velocities between 35 and $50 \mathrm{~cm} / \mu \mathrm{s}$. The timing uncertainty between the two systems was approximately $1 \mathrm{~ns}$, and each spectrum was integrated over \pm 5 ns from the plotted point. 
These data were used to infer the apparent plasma closure velocity

$$
\begin{array}{r}
v_{\text {eff }}=\frac{r_{2}-r_{1}}{t_{2}-t_{1}}=\frac{4.5[\mathrm{~mm}]}{t_{2}-t_{1}}, \\
n_{1}\left(t=t_{1}\right)=n_{2}\left(t=t_{2}\right)=n_{0},
\end{array}
$$

where $r_{1}$ and $r_{2}$ are the radial positions of fibers 1 and 2 with respect to the machine axis, respectively, $n_{1}(t)$ and $n_{2}(t)$ are the electron densities inferred from the data sets collected by probe one and two, respectively, and $n_{0}$ is a representative electron density, which was chosen to be between $2 \times 10^{17}$ and $4 \times 10^{17} \mathrm{~cm}^{-3}$. Fiber 1 is defined to be the probe closer to the machine axis, which is also the probe closer to the cathode. The plasma closure velocity in the SS-array experiment ranged from 45 to $50 \mathrm{~cm} / \mu \mathrm{s}$, and in the shaped-pulse liner experiment the velocity ranged from 35 to $41 \mathrm{~cm} / \mu \mathrm{s}$, depending on the value chosen for $n_{0}$. In both cases the velocity was over an order of magnitude above the expected thermal velocity of the plasma. Note however that the experimental value is similar to the $20 \mathrm{~cm} / \mu \mathrm{s}$ convolute-plasma closure velocities observed in simulations [22] and to the estimated $21 \mathrm{~cm} / \mu \mathrm{s}$ anode-plasma closure velocity assumed to explain impedance collapse in previous experiments [18].

The above measurements indicate that the plasma traveled from cathode to anode with a relatively high velocity. A probe was designed to verify this by observing a Doppler shift when viewing radially from the post to the cathode, as shown in Fig. 16. From the axial measurements,

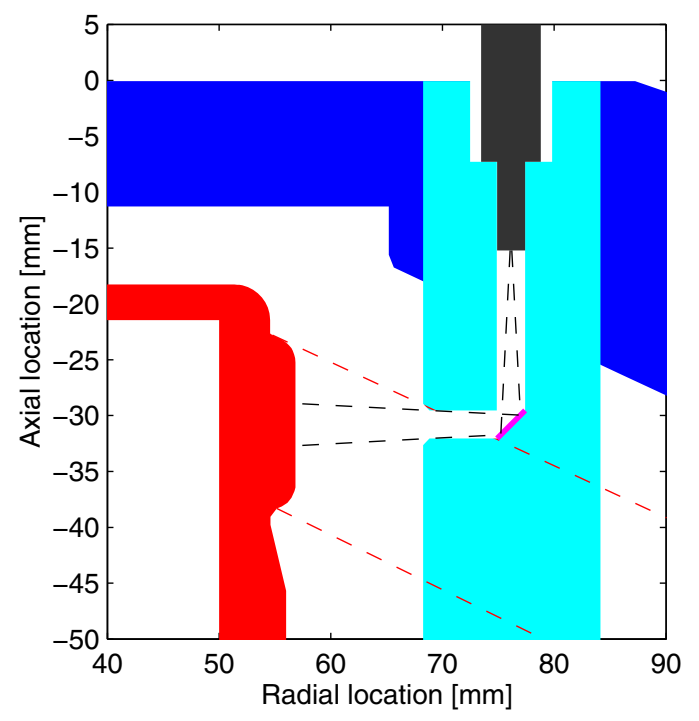

FIG. 16. An $R-Z$ cross section showing the radial view from post (anode) to cathode of the in-post convolute-spectroscopy probe. The surface shown in magenta was diamond turned to a mirror finish. The black dashed lines indicate the probe line of sight. the inferred plasma velocity would be towards the probe, thus the absorption feature would experience a Doppler shift to higher energy (blue shift). This assumes the continuum emission source was static (i.e., dense plasma at the electrode surface) and the source of absorption was moving. The Doppler shift of a spectral line is given by

$$
\Delta \lambda=\lambda_{0}\left(1-\sqrt{\frac{1+\frac{v}{c}}{1-\frac{v}{c}}}\right),
$$

where $\Delta \lambda$ is the wavelength shift, $\lambda_{0}$ is the wavelength of the spectral feature at zero velocity, $v$ is the velocity of the plasma in the direction of the probe, and $c$ is the speed of light. Given the $35-50 \mathrm{~cm} / \mu$ s plasma closure velocity, the expected shift in the $\mathrm{H} \alpha$ line was -7.0 to $-10.9 \AA$. The SVS diagnostic had a spectral accuracy of approximately $1 \AA$, so a multi-Å shift would be easily diagnosable. The measured $\mathrm{H} \alpha$ absorption feature was shifted to shorter wavelength by $1.2 \AA$, as shown in Fig. 17, which indicates the plasma velocity towards the probe was approximately $5.5 \mathrm{~cm} / \mu$ s. Given the $1 \AA$ wavelength uncertainty inherent in the SVS measurement, the range of possible plasma velocities was $5.5 \pm 4.5 \mathrm{~cm} / \mu$ s towards the anode post.

This result appears to contradict the data sets shown in Fig. 15; however, there is a hypothesis consistent with both data sets. Simulations indicate that plasma forms all over the convolute cathode surface; the plasma preferentially flows azimuthally along magnetic field lines and accumulates in the cathode hole, aligned azimuthally with the upper convolute post [22]. Rather than a dense plasma moving towards the anode at a high velocity, low-density

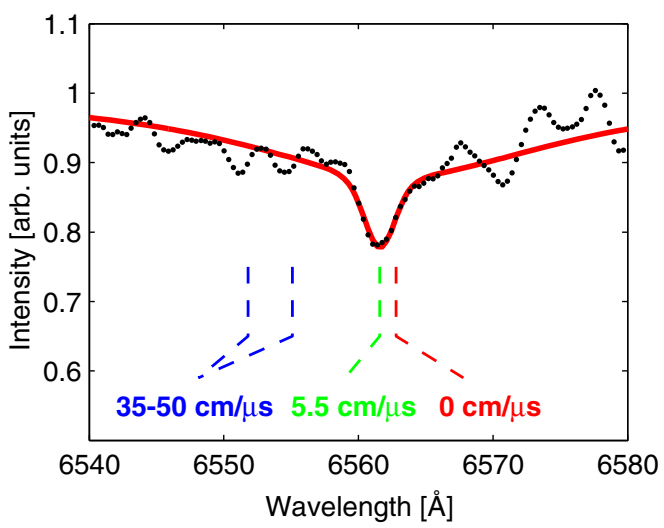

FIG. 17. A plot of the convolute plasma spectrum (black dots) using the probe specified in Fig. 16 at approximately $10 \mathrm{~ns}$ prior to stagnation ( $t=37 \mathrm{~ns}$ ). A fit to the data is shown in red. This fit indicates a $1.2 \AA$ shift to lower wavelength, which is consistent with a $5.5 \mathrm{~cm} / \mu$ s plasma moving towards the probe, assuming a stationary continuum source at the electrode surface. The accuracy of the wavelength scale is roughly $\pm 1 \AA$. Dashed vertical lines indicate where the center of the neutral hydrogen line would be for $0,5.5,35$, and $50 \mathrm{~cm} / \mu \mathrm{s}$, moving from right to left. 
plasma streams in azimuthally, creating a virtual motion of the plasma electron density towards the anode much faster than the average radial velocity of any particle in the plasma (see Fig. 2). The plasma cathode is still moving towards the anode at a rapid rate, but the individual particles are not, thus the motion is referred to as the apparent closure velocity. In this scenario, particle motion is normal to both radial and axial lines of sight, so a Doppler shift would not be observed with the axial or the radial probe.

Note that all spectroscopic measurements to date were made on the downstream side of the convolute. Previous simulations indicated that plasma collected in this area [22], so it was a logical place to make the initial measurements. It could be very informative to compare the plasma density histories at a few different locations in the convolute. Additional fields of view could help confirm or refute the hypothesis that plasma forming on the cathode streams along field lines and collects on the downstream side of the convolute. Such measurements will be the focus of future work.

The convolute impedance was estimated using a modified version of the Child-Langmuir law for space-charge limited emission,

$$
\begin{aligned}
Z_{\mathrm{calc}} & =\frac{9}{4} \sqrt{\frac{m}{2 e * V}} \frac{d^{2}}{A * \varepsilon_{0}}, \\
d & =d_{0}-v_{\mathrm{eff}} * \tau,
\end{aligned}
$$

where $A$ is the area of the emission, $\varepsilon_{0}$ is the permittivity of free space, $e$ is the electron charge, $m$ is the electron mass, $V$ is the applied voltage, $d_{0}=11.4 \mathrm{~mm}$ is the initial anodecathode distance, $v_{\text {eff }} \approx 40 \mathrm{~cm} / \mu$ s is the effective closure velocity, and $\tau$ is the time since plasma closure started. According to Eq. (7), the shunt impedance drops rapidly with increasing time. Note that even as the gap shrinks, the large magnetic field (tens of Tesla) is sufficient to insulate electrons in this region of the convolute. However, due to the complicated magnetic-field topology in this region, electrons emitted from the post-hole region can be lost to the anode at the transition to a radial transmission line downstream of the convolute.

Given the range of effective closure velocities $(35-50 \mathrm{~cm} / \mu \mathrm{s})$ and the anode-cathode gap on the downstream side of the upper post hole $(11.4 \mathrm{~mm})$, the approximate gap closure time was between 23 and $33 \mathrm{~ns}$. In the majority of the experiments investigated, stagnation of the z-pinch load occurred more than $50 \mathrm{~ns}$ after the convolute impedance collapse began. Assuming constant closure velocity, a plasma short would have existed for approximately $15-30 \mathrm{~ns}$ in the convolute on many experiments. The impedance of the convolute plasma was estimated by

$$
R=\frac{1}{N} * \frac{L}{\sigma A},
$$

where $N$ is the number of plasma shorts in parallel (assumed to be 12, one for each upper post hole), $A$ is the cross-sectional area of the plasma (assumed to be $1 \mathrm{~cm}^{2}$ ), $L$ is the length of the plasma (assumed to be $11.4 \mathrm{~mm}$ ), and $\sigma$ is the plasma conductivity. Assuming Spitzer conductivity

$$
\sigma=\frac{\left(4 \pi \varepsilon_{0}\right)^{2}\left(k_{B} T\right)^{3 / 2}}{\pi Z e^{2} m^{1 / 2} \ln (\Lambda)},
$$

where $k_{B}$ is Boltzmann's constant, $T=2 \mathrm{eV}$ is the plasma temperature, $Z=1$ is the effective ion charge state, and $\ln (\Lambda)$ is the Coulomb logarithm (approximately equal to 4 for these plasma parameters), the expected shunt impedance was $0.01 \mathrm{ohms}$.

There is a clear discrepancy between the calculated value (0.01 ohms) and the late-time impedance observed in experiments (typically $0.2-0.4 \mathrm{ohms}$ ). It is unlikely that the combined area of the 12 plasma bridges was 20-40 times smaller than estimated in this calculation, or that the plasma length was 20-40 times larger. Spitzer conductivity should be valid for these plasma conditions, so the most probable explanation for the discrepancy is that a static plasma bridge between the anode and cathode was never formed on the time scale of interest. A possible explanation as to why a stable, static plasma short cannot form is that the current flowing through such a short would be sufficiently high that the $j x B$ force on the plasma would accelerate/disrupt the connection. Any shorts that formed could rapidly clear and subsequently reform, so when averaged across all of the post holes, the effective convolute impedance would remain much higher than the value calculated above. If such a mechanism were present, it would also act to inject low-density plasma from the convolute into the inner-MITL, which might further compromise current delivery downstream of the inner-MITL B-dot monitors. Direct measurements of the environment in the inner-MITL would greatly help in understanding how plasma may be dynamically redistributed throughout these systems.

\section{CONCLUSIONS}

A measurable difference is observed between current at the vacuum insulator stack and current at the inner-MITL on most $Z$ machine experiments. Losses between the positions of these current measurements are primarily attributed to the double post-hole convolute due to the negligible losses observed between the stack and outerMITL B-dot locations. Previous simulations have shown that spaced-charge-limited emission at the magnetic nulls in the convolute cannot account for the observed losses and 
indicate that plasma formation in the convolute contributes substantially to the observed current loss [22,25-28].

The effective impedance of the convolute collapsed from greater than $10 \mathrm{ohms}$ to less than $1 \mathrm{ohm}$ over a roughly $10 \mathrm{~ns}$ period, and the impedance approached a relatively constant value of approximately $0.2-0.4 \mathrm{ohms}$. This behavior was observed across a wide variety of experiment types including those that are considered well matched to the driver, such as the $z$-pinch dynamic hohlraum. The variation in timing of the impedance collapse between different types of experiments is still under investigation.

Given the similar impedance behavior across many load types, coupled with a similar timing and magnitude of observed plasma density as a function of time, it appears that the wide range of observed shunt currents were primarily driven by the wide range of voltages applied to the convolute, which strongly depended on the load inductance history. Loads with high initial inductance and loads with high implosion velocities applied a larger voltage to the convolute, which caused a higher shunt current.

Spectroscopic measurements in the convolute show a dense plasma (greater than $1 \times 10^{16} \mathrm{~cm}^{-3}$ ) formed on the downstream side of the upper post hole for all types of experiments. The time of plasma formation appears correlated with the time of convolute impedance collapse. Simulations indicate that plasma formation in this region can contribute significantly to current loss.

The convolute plasma had an apparent cathode-to-anode velocity of approximately $35-50 \mathrm{~cm} / \mu \mathrm{s}$, which was much faster than expected for a roughly $2 \mathrm{eV}$ plasma. An estimate of the radially outward plasma velocity using Doppler spectroscopy suggested that this was not the velocity of charged particles in the plasma, but an effective bulk motion of the plasma. This is consistent with the hypothesis that low-beta plasma slowly streams in along magnetic field lines and collects downstream of the post, producing an apparent motion of the plasma across the gap between the cathode and the anode. Even with the rapid closure velocity, the impedance of the convolute did not drop below $0.1 \mathrm{ohms}$, indicating that a static plasma short across the post-hole gap did not form during the experiment.

Despite the progress made in diagnosing convolute plasma behavior, there are many outstanding questions. The formation time of a plasma electrode with sufficient density to support space-charge-limited emission $\left(10^{14} \mathrm{~cm}^{-3}\right)$, the correlation between high-density observations late in time and low-density behavior early in time, and the direction and velocity of plasma motion are still uncertain. Additionally, it is not clear by what process the neutral contaminant species are accelerated into the anodecathode gap, and the source/location of the continuum backlighter is in question. Additional investigations to answer these questions are planned and will be discussed in a future publication.
In light of the findings detailed above, the inner-MITL and load regions are being redesigned for a number of experiments to reduce the initial inductance. For example, the initial inductance for some upcoming magnetized liner inertial fusion experiments [7] was reduced from 6.5 to $4.6 \mathrm{nH}$, which should drop the peak convolute voltage from over 2.5 MV to less than 2 MV. This change is predicted to increase the current from 17-18 MA to 19-20 MA [43].

Additionally, a larger-diameter convolute was designed in order to improve current delivery to the load on $Z$ [64]. The new convolute was tested on several experiments, and preliminary results indicate that current delivery was improved. The new convolute design moved the posts radially outward, which decreased current density and reduced plasma formation in the post-hole region. Moving the posts to a larger radius also provided enough space to enlarge the holes in the cathode, which created larger A-K gaps for the plasma to cross. This comes at the cost of increased inductance inside the convolute, which increases the voltage applied to the convolute. Details of the new convolute will be the topic of a future publication.

\section{ACKNOWLEDGMENTS}

The authors acknowledge a series of extremely useful discussions on this topic with J. E. Bailey, J. Reneker, and G. A. Rochau. The authors would like to thank W.E. Fowler, J. K. Moore, M. E. Savage, and T. C. Wagoner for their work calibrating the electrical probes, D.E. Bliss, A. L. Carlson, D. H. Dolan, R. E. Falcon, R. G. Hacking, D. J. Scoglietti, C. T. Seagle, and C. C. Smith for their contributions to the fielding and calibration of the streakedvisible-spectroscopy systems, and the rest of the $\mathrm{Z}$ machine operations team for executing the experiments. The authors would also like to thank D. J. Ampleford, J. E. Bailey, E. C. Harding, B. Jones, M. C. Jones, G. P. Loisel, G. A. Rochau, and D. B. Sinars, who were the principal investigators (in addition to some of the authors) that allowed these spectroscopic, nonperturbative, ride-along measurements to occur on more than 50 experiments over six years. In addition, the authors thank E. A. Madrid, D. R. Welch, J. Gansz-Torres, and C. Mostrom for discussions related to the previously published numerical-simulation results. Portions of this research were funded by the National Nuclear Security Administration through the Krell Institute via the Stewardship Science Graduate Fellowship. This project was also funded in part by Sandia's Laboratory Directed Research and Development Program. Sandia National Laboratories is a multi-program laboratory managed and operated by Sandia Corporation, a wholly owned subsidiary of Lockheed Martin Corporation, for the U.S. Department of Energy's National Nuclear Security Administration under Contract No. DE-AC04-94AL85000. 
TABLE II. A list of the required time shifts in ns and the scaling factors that were applied to the inner-MITL currents to match the losslessly propagated stack currents for a series of short-circuit experiments.

\begin{tabular}{lclrr}
\hline \hline Shot \# & Date & Type & Shift & Scale \\
\hline 2224 & $7 / 12 / 11$ & Long & 0.6 & 0.964 \\
2225 & $7 / 13 / 11$ & Long & -1.2 & 0.939 \\
2266 & $11 / 23 / 11$ & Medium & 0.8 & 0.954 \\
2280 & $1 / 10 / 12$ & Short & 3.2 & 0.994 \\
2313 & $3 / 7 / 12$ & Short & 3.2 & 0.994 \\
2457 & $1 / 17 / 13$ & Shaped & -0.2 & 0.952 \\
2458 & $1 / 18 / 13$ & Standard & 2.0 & 0.952 \\
2499 & $3 / 29 / 13$ & Medium & 1.8 & 0.954 \\
2505 & $4 / 22 / 13$ & Standard & 2.0 & 0.958 \\
2546 & $9 / 5 / 13$ & Long & -1.2 & 0.937 \\
2569 & $10 / 23 / 13$ & Standard & 1.8 & 0.952 \\
2598 & $1 / 27 / 14$ & Short & 3.2 & 0.965 \\
2663 & $6 / 2 / 14$ & Standard & 2.4 & 0.968 \\
2894 & $1 / 5 / 16$ & Standard & 0.4 & 0.923 \\
2897 & $1 / 18 / 16$ & Standard & 0.7 & 0.940 \\
\hline \hline
\end{tabular}

\section{APPENDIX A: SHORT-CIRCUIT EXPERIMENTS}

Short-circuit experiments with low initial inductance were conducted on the $Z$ Machine to understand the electrical performance of different components in the system under a variety of electrical pulse shapes. The low-inductance, nonimploding loads applied very little electrical stress to the convolute, so minimal losses were expected. As a result, the losslessly propagated stack current and the inner-MITL current should have been equal; however, in all of these experiments the innerMITL current exceeded the losslessly propagated stack current, which is nonphysical. In order to get the signals to match, a scaling factor ranging from 0.923 to 0.994 and a time shift ranging from $-1.2 \mathrm{~ns}$ to $3.2 \mathrm{~ns}$ was required. On average, the scaling factor was $0.956 \pm 0.019$ and the temporal shift was $1.3 \pm 1.5 \mathrm{~ns}$. Details of the required scaling factors and time shifts are given in Table II.

\section{APPENDIX B: DETAILS ON THE STREAKED- VISIBLE-SPECTROSCOPY DIAGNOSTIC}

Each streaked-visible-spectroscopy system consisted of a 1 meter McPherson scanning monochromator (model 2061) and an NSTec L-CA-24 streak camera. The monochromator had a magnification of 1 , so the size of the fiber optic set the minimum spot size on the streak camera photocathode. The spectra were recorded on TMax 400 film using a microchannel plate with approximately $50 \mu \mathrm{m}$ effective pixel size. The signal was digitized using a microdensitometer with $22 \mu \mathrm{m}$ resolution. The resolution of the system was limited by the size of the fiber optic.
The fibers were high-OH silica multimode step-index fibers with a 0.22 numerical aperture. Measurements were collected using either a 100 or $200 \mu \mathrm{m}$ diameter core. The total fiber length was approximately $60 \mathrm{~m}$ split into three sections. A disposable 3 to $4 \mathrm{~m}$ fiber was used in the vacuum chamber, a 4 to $5 \mathrm{~m}$ fiber was embedded in a vacuum feedthrough, and a roughly $50 \mathrm{~m}$ fiber transmitted the signal to the diagnostics. Typical fiber transmission was approximately $35 \%$ at $4000 \AA, 45 \%$ at $5000 \AA$, and $50 \%$ at $6000 \AA$.

The spectrometers were fielded either with a survey grating $\left(\lambda_{\text {range }}=2600 \AA\right.$, $\left.\lambda_{\text {resolution }}=10 \AA\right)$ or with a highresolution grating $\left(\lambda_{\text {range }}=650 \AA, \lambda_{\text {resolution }}=2.7 \AA\right)$. The spectral scale was determined for each data set collected with the survey grating by exposing a set of laser lines of known wavelength on the image. The accuracy of the spectral scale was approximately $1.5 \AA$ for the survey grating based on the wavelength per pixel of the digitized spectrum. In high-resolution measurements, only one absolute wavelength fiducial was within the spectral range, so the spectral scale was determined using a separate calibration measurement with a Xe flash lamp. The scale determined using the Xe flash lamp was applied to the experimental measurement using the same laser line on both spectra as an absolute reference. For the highresolution measurements, the wavelength-scale accuracy was approximately $1 \AA$, based primarily on the accuracy with which the diffraction grating was positioned between the calibration and the measurement (a manually positioned knob with $2 \AA /$ tick could be set to \pm 0.5 ticks). The spectral resolution of the system for a particular setup was determined using the spectral FWHM of the laser lines on the digitized spectrum.

The streak cameras covered a roughly $220 \mathrm{~ns}$ window with approximately $2 \mathrm{~ns}$ temporal resolution or a roughly $440 \mathrm{~ns}$ window with $4 \mathrm{~ns}$ resolution. The temporal resolution of each system was determined by injecting a $0.1 \mathrm{~ns}$ FWHM light pulse into the collection fiber and measuring the temporal FWHM of the recorded signal. Typically, the experimental signals were integrated over 5-10 ns windows to increase the signal-to-noise ratio, so the temporal resolution of the diagnostic did not limit these measurements. The cross timing between the two SVS diagnostics was approximately $1 \mathrm{~ns}$ and was dominated by uncertainty in the time of flight of the approximately $60 \mathrm{~m}$ long fiber chain. Cross timing between the systems could be improved to sub-ns accuracy with limited effort but has not been a priority due to the relatively long signal-integration times. The cross timing between digitizer traces on $Z$ is typically quoted at $1 \mathrm{~ns}$; however, Appendix A showed that there is a combined systematic-plus-random uncertainty of approximately $2 \mathrm{~ns}$ between the different electrical monitors, so the cross timing between the optical and electrical signals was likely within 2 to $3 \mathrm{~ns}$. 

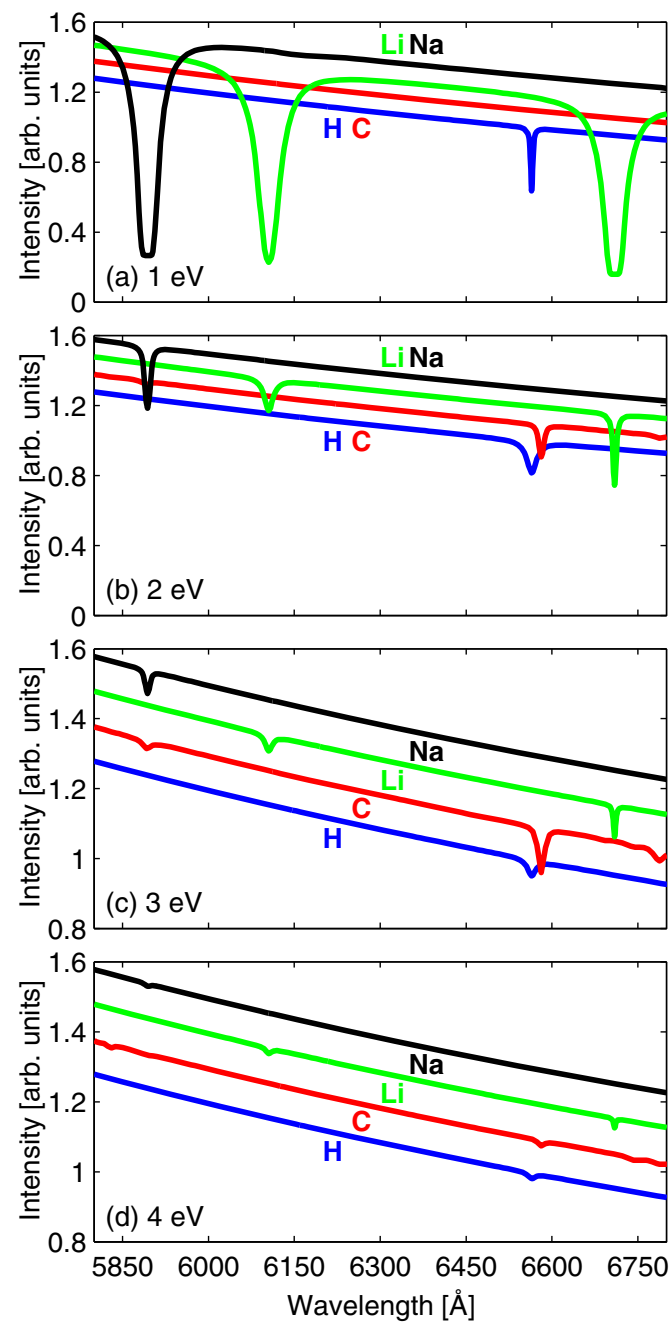

FIG. 18. (a) Plots of the hydrogen, carbon, lithium, and sodium spectra generated with PRISMSPECT with an ion density of $2 \times 10^{17} \mathrm{~cm}^{-3}$ and an electron temperature of $1 \mathrm{eV}$. The experimentally observed singly ionized carbon lines at 6578 and $6583 \AA$ are not observed in the simulated spectra. (b) Plots of the spectra at $2 \mathrm{eV}$. All experimentally observed features are also observed in the simulated spectra. (c) Plots of the spectra at $3 \mathrm{eV}$. All of the experimentally observed features are observed in the simulated spectra, but the neutral sodium and hydrogen lines are just below the assigned 5\% limit of detectability. (d) Plots of the spectra at $4 \mathrm{eV}$. All of the spectral features are below the assigned $5 \%$ limit of detectability. Note that the $\mathrm{H}, \mathrm{Li}$, and $\mathrm{Na}$ plots were offset by $-0.1,+0.1$, and +0.2 for clarity.

\section{APPENDIX C: PRISMSPECT SIMULATIONS}

A series of PRISMSPECT [56] simulations were conducted to determine likely plasma parameters given the experimentally observed spectral features. The simulations were run using a single element $(\mathrm{H}, \mathrm{Li}, \mathrm{C}$, or $\mathrm{Na})$ in nonlocal thermodynamic equilibrium (non-LTE). The simulations used a planar geometry ranging from $0.1 \mathrm{~mm}$ (highly localized electrode plasma) to $10 \mathrm{~mm}$ thick (plasma spanning the anode-cathode gap). All experimental measurements included a continuum-emission source with absorption features, so the simulations used a continuum backlight with a Planckian temperature ranging from 1 to $10 \mathrm{eV}$. The ion density was varied from $1 \times 10^{15}$ to $1 \times 10^{18} \mathrm{~cm}^{-3}$ to cover the range of inferred densities from the experiments, and the plasma temperature ranged from 0.1 to $10 \mathrm{eV}$ to more than cover the span of MITL plasma temperatures reported in the literature.

The simulated $\mathrm{H}$ spectra were scanned to identify the plasma conditions where the line at $6563 \AA$ was present but the line at $4861 \AA$ was absent. Due to noise in the experimental spectra, lines could only be readily identified if the amplitude of absorption exceeded approximately $5 \%$ of the local continuum. Thus, $5 \%$ was used as a threshold to determine when lines would be observed in the simulated spectra. Based on this analysis, the plasma thickness appeared to be greater than or equal to $5 \mathrm{~mm}$, and the temperature of the backlight was not critical as long as it was greater than $4 \mathrm{eV}$. The simulations with $\mathrm{Li}, \mathrm{C}$, and $\mathrm{Na}$ were run with a $5 \mathrm{~mm}$ thickness and a $7 \mathrm{eV}$ backlight.

The range of densities consistent with the Stark broadening measurements over the majority of the experiments was up to $4 \times 10^{17} \mathrm{~cm}^{-3}$. Given this density range, the typical $\mathrm{H}$ spectrum was well reproduced by plasma temperatures between 0.7 and $3 \mathrm{eV}$, the observed $\mathrm{Li} \mathrm{I}$ line required temperatures from 0.1 to $3.5 \mathrm{eV}$, the $\mathrm{C}$ II lines required 2 to $3.5 \mathrm{eV}$, and the $\mathrm{Na}$ I feature required 0.1 to $2.5 \mathrm{eV}$. The full temperature range that could produce one or more of the observed lines is $0.1-3.5 \mathrm{eV}$, and the range consistent with all of the spectral features is $2-2.5 \mathrm{eV}$. These simulations, coupled with the observed spectral features, indicate that temperatures in different regions of the plasma likely spanned from less than $1 \mathrm{eV}$ to several eV. Plots of the calculated spectra at a few temperatures are shown in Fig. 18.

[1] M. E. Savage, K. R. LeChien, M. R. Lopez, B. S. Stoltzfus, W. A. Stygar, D. S. Artery, J. A. Lott, and P. A. Corcoran, Status of the $Z$ pulsed power driver, in Proceedings of the 18th International Pulsed Power Conference, Chicago, Illinois (IEEE, New York, 2011), pp. 983-990, http:// ieeexplore.ieee.org/document/6191629/?reload=true.

[2] D. V. Rose, D. R. Welch, E. A. Madrid, C. L. Miller, R. E. Clark, W. A. Stygar, M. E. Savage, G. A. Rochau, J. E. Bailey, T. J. Nash, M. E. Sceiford, K. W. Struve, P. A. Corcoran, and B. A. Whitney, Three-dimensional electromagnetic model of the pulsed-power Z-pinch accelerator, Phys. Rev. ST Accel. Beams 13, 010402 (2010).

[3] J. P. Davis, C. Deeney, M. D. Knudson, R. W. Lemke, T. D. Pointon, and D. E. Bliss, Magnetically driven isentropic compression to multimegabar pressures using shaped current pulses on the $Z$ accelerator, Phys. Plasmas 12, 056310 (2005). 
[4] M. R. Martin, R. W. Lemke, R. D. McBride, J. P. Davis, D. H. Dolan, M. D. Knudson, K. R. Cochrane, D. B. Sinars, I. C. Smith, M. Savage, W. A. Stygar, K. Killebrew, D. G. Flicker, and M. C. Herrmann, Solid liner implosions on $Z$ for producing multi-megabar, shockless compressions, Phys. Plasmas 19, 056310 (2012).

[5] M. E. Cuneo et al., Development and characterization of a $Z$-pinch-driven hohlraum high-yield inertial confinement fusion target concept, Phys. Plasmas 8, 2257 (2001).

[6] R. D. McBride et al., Penetrating Radiography of Imploding and Stagnating Beryllium Liners on the $Z$ Accelerator, Phys. Rev. Lett. 109, 135004 (2012).

[7] M. R. Gomez et al., Experimental Demonstration of Fusion-Relevant Conditions in Magnetized Liner Inertial Fusion, Phys. Rev. Lett. 113, 155003 (2014).

[8] G. A. Rochau, J. E. Bailey, R. E. Falcon, G. P. Loisel, T. Nagayama, R. C. Mancini, I. Hall, D. E. Winget, M. H. Montgomery, and D. A. Leidahl, ZAPP: The $Z$ astrophysical plasma properties collaboration, Phys. Plasmas 21, 056308 (2014).

[9] J. E. Bailey et al., A higher-than-predicted measurement of iron opacity at solar interior temperatures, Nature (London) 517, 56 (2014).

[10] R. B. Spielman, P. Corcoran, J. Fockler, H. Kishi, and P.W. Spence, A double post-hole convolute diode for $Z$-pinch experiments on Saturn, in Proceedings of the 7th International Pulsed Power Conference, Monterey, California 1989 (IEEE, New York, 1989), pp. 445-448, http://ieeexplore.ieee.org/document/767519/.

[11] T. D. Pointon, W. A. Stygar, R. B. Spielman, H. C. Ives, and K.W. Struve, Particle-in-cell simulations of electron flow in the post-hole convolute of the $\mathrm{Z}$ accelerator, Phys. Plasmas 8, 4534 (2001).

[12] T. W. L. Sanford, R. E. Olson, R. L. Bowers, G. A. Chandler, M. S. Derzon, D. E. Hebron, R. J. Leeper, R. C. Mock, T. J. Nash, D. L. Peterson, L. E. Ruggles, W. W. Simpson, K. W. Struve, and R. A. Vesey, Z-PinchGenerated $X$ Rays Demonstrate Potential for IndirectDrive ICF Experiments, Phys. Rev. Lett. 83, 5511 (1999).

[13] J. E. Bailey et al., Dynamic hohlraum radiation hydrodynamics, Phys. Plasmas 13, 056301 (2006).

[14] D. D. Bloomquist, R. W. Stinnett, D. H. McDaniel, J. R. Lee, A. W. Sharpe, J. A. Halbleib, L. G. Schlitt, P. W. Spence, and P. Corcoran, SATURN, A large area $\mathrm{x}$-ray simulation accelerator, in Proceedings of the 6th International Pulsed Power Conference, Arlington, Virginia, 1987 (IEEE, New York, 1987), pp. 310-317.

[15] K. W. Struve, T. H. Martin, R. B. Spielman, W. A. Stygar, P. A. Corcoran, and J. W. Douglas, Circuit-code modeling of the PBFA $\mathrm{Z}$ for $\mathrm{Z}$-pinch experiments, in Proceedings of the 11th International Pulsed Power Conference, Baltimore, Maryland (IEEE, Piscataway, NJ, 1997), pp. 162167, http://ieeexplore.ieee.org/document/679300/.

[16] D. L. Peterson, R. L. Bowers, W. Matuska, K. D. McLenithan, G. A. Chandler, C. Deeney, M. S. Derzon, M. Douglas, M. K. Matzen, T. J. Nash, R. B. Spielman, K. W. Struve, W. A. Stygar, and N. F. Roderick, Insights and applications of two-dimensional simulations to Z-pinch experiments, Phys. Plasmas 6, 2178 (1999).
[17] R. W. Lemke, M. D. Knudson, A. C. Robinson, T. A. Haill, K. W. Struve, J. R. Asay, and T.A. Mehlhorn, Self-consistent, two-dimensional, magnetohydrodynamic simulations of magnetically driven flyer plates, Phys. Plasmas 10, 1867 (2003).

[18] C. A. Jennings, J. P. Chittenden, M. E. Cuneo, W. A. Stygar, D. J. Ampleford, E. M. Waisman, M. Jones, M. E. Savage, K. R. LeChien, and T. C. Wagoner, Circuit model for driving three-dimensional resistive MHD wire array Z-pinch calculations, IEEE Trans. Plasma Sci. 38, 529 (2010).

[19] C. A. Jennings, M. E. Cuneo, E. M. Waisman, D. B. Sinars, D. J. Ampleford, G. R. Bennett, W. A. Stygar, and J. P. Chittenden, Simulations of the implosion and stagnation of compact wire arrays, Phys. Plasmas 17, 092703 (2010).

[20] M. R. Gomez, M. E. Cuneo, J.-P. Davis, R. W. Lemke, R. D. McBride, R. B. Campbell, C. A. Jennings, W. A. Stygar, D. V. Rose, D. R. Welch, and E. A. Madrid, A systematic study of current flow and impedance behavior in the $Z$ machine double post-hole convolute, in Proceedings of the 19th International Pulsed Power Conference, San Francisco, California (IEEE, New York, 2013), pp. 63-68, http://ieeexplore.ieee.org/document/6627397/.

[21] B. Jones et al., A renewed capability for gas puff science on Sandia's Z machine, IEEE Trans. Plasma Sci. 42, 1145 (2014).

[22] D. V. Rose, D. R. Welch, T. P. Hughes, R. E. Clark, and W. A. Stygar, Plasma evolution and dynamics in highpower vacuum-transmission-line post-hole convolutes, Phys. Rev. ST Accel. Beams 11, 060401 (2008).

[23] T. D. Pointon, W. L. Langston, and M. E. Savage, Computer simulations of the magnetically insulated transmission lines and post-hole convolute of $Z R$, in Proceedings of the 16th IEEE International Pulsed Power Conference, Albuquerque, NM (IEEE, Piscataway, NJ, 2007), pp. 165-170, http://ieeexplore.ieee.org/document/ 4651814/.

[24] D. V. Rose, D. R. Welch, T. P. Hughes, R. E. Clark, C. B. Mostrom, and W. A. Stygar, Plasma formation, evolution, and dynamics in 100 TW vacuum-transmission-line posthole convolutes, in Proceedings of the 16th IEEE International Pulsed Power Conference, Albuquerque, NM (IEEE, Piscataway, NJ, 2007), pp. 171-174, http:// ieeexplore.ieee.org/document/4651815/.

[25] D. V. Rose, D. R. Welch, R. E. Clark, E. A. Madrid, C. L. Miller, C. Mostrom, W. A. Stygar, M. E. Cuneo, C. A. Jennings, B. Jones, D. J. Ampleford, and K. W. Struve, $Z R$-convolute analysis and modeling: plasma evolution and dynamics leading to current losses, in Proceedings of the 17th International Pulsed Power Conference, Washington, DC (IEEE, New York, 2009), pp. 11531158, http://ieeexplore.iee.org/document/5386268/.

[26] T. D. Pointon and D. B. Seidel, Current loss in the vacuum section of the refurbished $Z$ accelerator, in Proceedings of the 17th International Pulsed Power Conference, Washington, DC (IEEE, New York, 2009), pp. 11591164, http://ieeexplore.iee.org/document/5386319/.

[27] E. A. Madrid, D. V. Rose, D. R. Welch, R. E. Clark, C. B. Mostrom, W. A. Stygar, M. E. Cuneo, M. R. Gomez, T. P. Hughes, T. D. Pointon, and D. B. Seidel, Steady-state 
modeling of current loss in a post-hole convolute driven by high power magnetically insulated transmission lines, Phys. Rev. ST Accel. Beams 16, 120401 (2013).

[28] D. V. Rose, E. A. Madrid, D. R. Welch, R. E. Clark, C. B. Mostrom, W. A. Stygar, and M. E. Cuneo, Computational analysis of current-loss mechanisms in a post-hole convolute driven by magnetically insulated transmission lines, Phys. Rev. ST Accel. Beams 18, 030402 (2015).

[29] J. P. VanDevender, R. W. Stinnett, and R. J. Anderson, Negative ion losses in magnetically insulated vacuum gaps, Appl. Phys. Lett. 38, 229 (1981).

[30] V. V. Alexandrov, E. A. Azizov, S. G. Alikhanov, E. V. Grabovsky, A. P. Lototsky, S. L. Nedoseev, G. M. Oleinik, V.P. Smirnov, V. A. Yagnov, A. M. Zhitlukhin, E. P. Velikhov, V. A. Glukhih, V. C. Kuchinsky, O. P. Pechersky, and P. V. Sasorov, Superfast liner implosion physics study and development of X-ray facility based on 900 MJ inductive store, in Proceedings of the 12th IEEE International Pulsed Power Conference (IEEE, Piscataway, NJ, 1999), pp. 754-757, http://ieeexplore .ieee.org/document/823623/.

[31] E. V. Grabovsky et al., Development of x-ray facility "Baikal" based on 900 MJ inductive store and related problems, in Proceedings of the 13th International Pulsed Power Conference, Las Vegas, Nevada (IEEE, New York, 2001), pp. 773-776, http://ieeexplore.ieee.org/ document/960788/.

[32] V. P. Bakhtin et al., Part testing of the "MOL" stand for the "Baikal" project, in Proceedings of the 15th International Pulsed Power Conference, Monterey, California (IEEE, New York, 2005), pp. 394-397, http://ieeexplore.ieee.org/ document/4084235/.

[33] W. A. Stygar, M. E. Cuneo, D. I. Headley, H. C. Ives, R. J. Leeper, M. G. Mazarakis, C. L. Olson, J. L. Porter, T. C. Wagoner, and J. R. Woodworth, Architecture of petawattclass z-pinch accelerators, Phys. Rev. ST Accel. Beams 10, 030401 (2007).

[34] W. A. Stygar et al., Conceptual designs of two petawattclass pulsed-power accelerators for high-energy-densityphysics experiments, Phys. Rev. ST Accel. Beams 18, 110401 (2015).

[35] T. C. Wagoner et al., Differential-output $B$-dot and $D$-dot monitors for current and voltage measurements on a 20MA, 3-MV pulsed-power accelerator, Phys. Rev. ST Accel. Beams 11, 100401 (2008).

[36] J. T. Crow and G. D. Peterson, Recovery of electron sheath current in magnetically self-insulated transmission lines, IEEE Trans. Plasma Sci. 11, 219 (1983).

[37] E. M. Waisman, M. E. Cuneo, R. W. Lemke, D. B. Sinars, and W. A. Stygar, Lower bounds for the kinetic energy and resistance of wire array $Z$ pinches on the $Z$ pulsed-power accelerator, Phys. Plasmas 15, 042702 (2008).

[38] R. D. McBride, C. A. Jennings, R. A. Vesey, G. A. Rochau, M. E. Savage, W. A. Stygar, M. E. Cuneo, D. B. Sinars, M. Jones, K. R. LeChien, M. R. Lopez, J. K. Moore, K. W. Struve, T. C. Wagoner, and E. M. Waisman, Displacement current phenomena in the magnetically insulated transmission lines of the refurbished $Z$ accelerator, Phys. Rev. ST Accel. Beams 13, 120401 (2010).
[39] E. M. Waisman, R. D. McBride, M. E. Cuneo, D. F. Wenger, W. E. Fowler, W. A. Johnson, L. I. Basilio, R. S. Coats, C. A. Jennings, D. B. Sinars, R. A. Vesey, B. Jones, D. J. Ampleford, R. W. Lemke, M. R. Martin, P. C. Schrafel, S. A. Lewis, J. K. Moore, M. E. Savage, and W. A. Stygar, Voltage measurements at the vacuum posthole convolute of the $\mathrm{Z}$ pulsed-power accelerator, Phys. Rev. ST Accel. Beams 17, 120401 (2014).

[40] W. A. Stygar et al., 55-TW magnetically insulated transmission-line system: Design, simulations, and performance, Phys. Rev. ST Accel. Beams 12, 120401 (2009).

[41] D. J. Ampleford et al., Contrasting physics in wire array $z$ pinch sources of $1-20 \mathrm{keV}$ emission on the $Z$, Phys. Plasmas 21, 056708 (2014).

[42] D. C. Rovang et al., Pulsed-coil magnet systems for applying uniform 10-30 T fields to centimeter-scale targets on Sandia's Z facility, Rev. Sci. Instrum. 85, 124701 (2014).

[43] B. T. Hutsel (private communication).

[44] J. E. Bailey, A. B. Filuk, A. L. Carlson, D. J. Johnson, P. Lake, E. J. McGuire, T. A. Mehlhorn, T. D. Pointon, T. J. Renk, W. A. Stygar, and Y. Maron, Measurements of Acceleration Gap Dynamics in a 20-TW AppliedMagnetic-Field Ion Diode, Phys. Rev. Lett. 74, 1771 (1995).

[45] A. B. Filuk, J. E. Bailey, A. L. Carlson, D. J. Johnson, P. Lake, T. A. Mehlhorn, L. P. Mix, T. J. Renk, W. A. Stygar, and Y. Maron, Charge-Exchange Atoms and Ion Source Divergence in a 20 TW Applied- $B$ Ion Diode, Phys. Rev. Lett. 77, 3557 (1996).

[46] J. E. Bailey, M. D. Knudson, A. L. Carlson, G. S. Dunham, M. P. Desjarlais, D. L. Hanson, and J. R. Asay, Timeresolved optical spectroscopy measurements of shocked liquid deuterium, Phys. Rev. B 78, 144107 (2008).

[47] M. R. Gomez, R. M. Gilgenbach, M. E. Cuneo, R. D. McBride, G. A. Rochau, D. J. Ampleford, J. E. Bailey, A. D. Edens, B. Jones, M. Jones, M. R. Lopez, M. E. Savage, D. B. Sinars, and W. A. Stygar, Spectroscopic measurements in the post-hole convolute on Sandia's $Z$-machine (invited), in Proceedings of the 18th International Pulsed Power Conference, Chicago, Illinois (IEEE, New York, 2011), pp. 688-695, http://ieeexplore .ieee.org/document/6191564/.

[48] R. B. King, Relative transition probabilities of the Swan bands of carbon, Astrophys. J. 108, 429 (1948).

[49] D. V. Rose, D. R. Welch, C. L. Miller, R. E. Clark, E. A. Madrid, C. B. Mostrom, T. C. Wagoner, J. K. Moore, W. A. Stygar, J. E. Bailey, T. J. Nash, G. A. Rochau, and D. B. Sinars, $10^{7}$-A load-current $B$-dot monitor: Simulations, design, and performance, Phys. Rev. ST Accel. Beams 13, 040401 (2010).

[50] M. E. Cuneo, P. R. Menge, D. L. Hanson, W. E. Fowler, M. A. Bernard, G. R. Ziska, A. B. Filuk, T. D. Pointon, R. A. Vesey, D. R. Welch, J. E. Bailey, M. P. Desjarlais, T. R. Lockner, T. A. Mehlhorn, S. A. Slutz, and M. A. Stark, Results of vacuum cleaning techniques on the performance of LiF field-threshold ion sources on extraction applied- $B$ ion diodes at $1-10$ TW, IEEE Trans. Plasma Sci. 25, 229 (1997). 
[51] R. W. Stinnett, G. R. Allen, H. P. Davis, T. W. Hussey, G. J. Lockwood, M. A. Palmer, L. E. Ruggles, A. Widman, H. N. Woodall, and R.D. Bengtson, Cathode plasma formation in magnetically-insulated transmission lines, IEEE Trans. Electr. Insul. EI-20, 807 (1985).

[52] M. Jones (private communication).

[53] M. R. Gomez, S. B. Hansen, K. J. Peterson, D. E. Bliss, A. L. Carlson, D. C. Lamppa, D. G. Schroen, and G. A. Rochau, Magnetic field measurements via visible spectroscopy on the Z machine, Rev. Sci. Instrum. 85, 11E609 (2014).

[54] M.E. Cuneo, The effect of electrode contamination, cleaning and conditioning on high-energy pulsed-power device performance, IEEE Trans. Dielectr. Electr. Insul. 6, 469 (1999).

[55] M. E. Cuneo, R. M. Gilgenbach, and M. L. Brake, Spectroscopic study of anode plasmas in a microsecond electron beam diode, IEEE Trans. Plasma Sci. 15, 375 (1987).

[56] http://www.prism-cs.com.

[57] V. V. Ivanov, P. J. Laca, B. S. Bauer, R. Presura, V. I. Sotnikov, A. L. Astanovitskiy, B. Le Galloudec, J. Glassman, and R. A. Wirtz, Investigation of plasma evolution in a coaxial small-gap magnetically insulated transmission line, IEEE Trans. Plasma Sci. 32, 1843 (2004).

[58] T. J. Awe, B. S. Bauer, S. Fuelling, I. R. Lindemuth, and R. E. Siemon, Experimental investigation of thermal plasma formation from thick aluminum surfaces by pulsed multimegagauss magnetic field, Phys. Plasmas 17, 102507 (2010).

[59] S. A. Chaikovsky, V. I. Oreshkin, I. M. Datsko, N. A. Labetskaya, and N. A. Ratakhin, Skin explosion of double-layer conductors in fast-rising high magnetic fields, Phys. Plasmas 21, 042706 (2014).

[60] M. A. Gigosos and V. Cardenoso, New plasma diagnosis tables of hydrogen Stark broadening including ion dynamics, J. Phys. B 29, 4795 (1996).

[61] C. Thoma, T. C. Genoni, D. R. Welch, D. V. Rose, R. E. Clark, C. L. Miller, W. A. Stygar, and M. L. Kiefer, Numerical simulation of cathode plasma dynamics in magnetically insulated vacuum transmission lines, Phys. Plasmas 22, 032101 (2015).

[62] K. J. Peterson, D. B. Sinars, E. P. Yu, M. C. Herrmann, M. E. Cuneo, S. A. Slutz, I. C. Smith, B. W. Atherton, M. D. Knudson, and C. Nakhleh, Electrothermal instability growth in magnetically driven pulsed power liners, Phys. Plasmas 19, 092701 (2012).

[63] R. W. Stinnett, M. A. Palmer, R. B. Spielman, and R. Bengtson, Small gap experiments in magnetically insulated transmission lines, IEEE Trans. Plasma Sci. 11, 216 (1983).

[64] D. C. Rovang (private communication). 